JOURNAL OF

FUNCTION SPACES AND APPLICATIONS

Volume 2, Number 1 (2004), 25-53
(C) 2004, Scientific Horizon http://www.jfsa.net

\title{
Spaces of Test Functions via the STFT
}

\author{
Karlheinz Gröchenig and Georg Zimmermann \\ (Communicated by Hans Feichtinger)
}

1991 Mathematics Subject Classification. 46F05, 81S30.

Keywords and phrases. Schwartz class, ultradistributions, short-time Fourier transform, Gelfand-Shilov spaces, modulation spaces, Gabor frame, logarithmic integral.

Abstract. We characterize several classes of test functions, among them Björck's ultra-rapidly decaying test functions and the Gelfand-Shilov spaces of type $S$, in terms of the decay of their short-time Fourier transform and in terms of their Gabor coefficients.

\section{Introduction}

The definition of the Fourier transform on the level of distributions is founded on a solid theory of spaces of test functions that are invariant under the Fourier transform.

In the standard theories, classes of test functions are defined by separate conditions on time and frequency. The typical example is the Schwartz class $\mathcal{S}$, where a member $\varphi \in \mathcal{S}$ is defined by the rapid decay of all derivatives $\mathrm{D}^{p} \varphi$ and $\mathrm{D}^{q} \widehat{\varphi}$. However, many applications, for instance the concept of phase space content in quantum mechanics, or the local frequency spectrum in signal analysis, require a joint time-frequency description of functions. This point of view motivated us to look closely at the joint time-frequency behavior of test functions and distributions. In the context of time-frequency 
analysis, such an investigation is carried out conveniently by means of the short-time Fourier transform and its relatives, such as the radar ambiguity function or the Wigner distribution. Earlier results in this direction have been obtained by Janssen [20] and Cho [5] for certain Gelfand-Shilov spaces and by the authors for the Schwartz class [17]. The investigation of certain small modulation spaces (Banach spaces contained in $\mathcal{S}$ ) and the duals in $[14,25,27]$ can also be seen in this context.

Our objective is a more comprehensive analysis of spaces of test functions and corresponding distributions. We will investigate the time-frequency behavior of functions in Björck's spaces of ultra-rapidly decaying test functions [1] and in the Gelfand-Shilov spaces or Gevrey classes [13, 18]. These classes of test functions are refinements of the Schwartz class and occur often in the theory of partial differential equations [18]. Our main result yields a characterization of these spaces by their joint time-frequency behavior (Theorems 2.7, 3.8 and 3.11). From the point of view of timefrequency analysis these characterizations are quite natural, though not yet established in analysis. To persuade the reader of the convenience and usefulness of these characterizations, we give three applications. Firstly, we will give a new proof of Kashpirovskij's theorem [22] which shows that the usual definition of spaces of test functions can be weakened and can be done solely by decay conditions without using derivatives (Cor. 2.9 and 3.9). Secondly, we give a significant improvement of Janssen's theorem on the window design for Gabor frames in Thm. 4.2. Thirdly, we then characterize ultra-rapidly decaying test functions by their Gabor coefficients.

The paper is organized as follows: In Section 2, we introduce Björck's ultra-rapidly decaying test functions and ultra-distributions, and investigate their joint time-frequency behavior by using the techniques developed in [17]. In Section 3, a similar program is carried out for the spaces of type $S$ (or Gevrey classes) of Gelfand and Shilov. Their characterization by means of the STFT is more challenging and will be given in Theorem 3.8 and Corollary 3.11. Finally, in Section 4 we present a different perspective on spaces of test functions in terms of so-called modulation spaces and give another characterization of ultra-rapidly decaying test functions by means of Gabor frames.

1.1. The short-time Fourier transformation (STFT). Our main tool for the investigation of test functions and distributions will be the short-time Fourier transform.

Definition. The short-time Fourier transform (STFT) of a function or distribution $f$ on $\mathbb{R}^{d}$ with respect to the non-zero window $g$ is defined as

$$
\left(\mathcal{V}_{g} f\right)(x, \xi)=\int_{\mathbb{R}^{d}} f(t) \overline{g(t-x)} e^{-2 \pi i \xi t} d t=\left(\widehat{f \overline{\mathrm{T}_{x} g}}\right)(\xi)=\left\langle f, \mathrm{M}_{\xi} \mathrm{T}_{x} g\right\rangle,
$$


where as usual $\mathrm{T}_{x} f(t)=f(t-x)$ is the translation operator and $\mathrm{M}_{\xi} f(t)=$ $e^{2 \pi i \xi t} f(t)$ is the modulation operator.

The quantity $\mathcal{V}_{g} f(x, \xi)$ is a measure for the amplitude of the frequency band near $\xi$ at time $x$. We will heavily use the following properties of the STFT.

Lemma 1.1. For functions $f, f_{i}, g, g_{i}$, and $h \in \mathcal{S}\left(\mathbb{R}^{d}\right)$, the $S T F T$ has the following properties.

(i) (Inversion formula)

$$
\iint_{\mathbb{R}^{d} \times \widehat{\mathbb{R}}^{d}}\left(\mathcal{V}_{g} f\right)(x, \xi) \mathrm{M}_{\xi} \mathrm{T}_{x} h d x d \xi=\langle h, g\rangle f .
$$

(ii) (STFT of the Fourier transforms)

$$
\left(\mathcal{V}_{\widehat{g}} \widehat{f}\right)(\eta, y)=e^{-2 \pi i \eta y}\left(\mathcal{V}_{g} f\right)(-y, \eta) .
$$

(iii) (Fourier transform of the STFT)

$$
\widehat{\left(\mathcal{V}_{g} f\right)}(\eta, y)=e^{2 \pi i \eta y} f(-y) \overline{\widehat{g}(\eta)} .
$$

The proofs are straightforward calculations and can be found, e.g., in $[14,17]$.

1.2. Notation. We use standard multi-index notation and write $x^{p}=$ $\left(x_{1}, \ldots, x_{d}\right)^{\left(p_{1}, \ldots, p_{d}\right)}=\prod_{i=1}^{d} x_{i}^{p_{i}}, D^{p}=\prod_{i=1}^{d} \frac{\partial^{p_{i}}}{\partial x_{i}^{p_{i}}}, \alpha \leq \beta \Longleftrightarrow \alpha_{i} \leq \beta_{i}, \forall i=$ $1 \ldots d$ and $\alpha, \beta \in \mathbb{N}_{0}^{d}$. Using the multinomial coefficients $\left(\begin{array}{l}p \\ r\end{array}\right)=\prod_{i=1}^{d}\left(\begin{array}{l}p_{i} \\ r_{i}\end{array}\right)$ yields $(a+b)^{p}=\sum_{r \leq p}\left(\begin{array}{c}p \\ r\end{array}\right) a^{r} b^{p-r}$. We shall denote the Euclidean norm by $\|x\|$ and the $\boldsymbol{\ell}^{1}$-norm by $\|x\|_{1}=\sum_{i=1}^{d}\left|x_{i}\right|$, while by the absolute value of a vector, we mean the vector of absolute values $|x|=\left(\left|x_{1}\right|, \ldots,\left|x_{d}\right|\right)$.

\section{Ultra-rapidly decaying test functions and tempered ultra-distributions}

2.1. Weight functions. In general, a weight function is simply a nonnegative, locally integrable function on $\mathbb{R}^{d}$.

Following [28], we define $\mathcal{M}$ to be the collection of all nonnegative functions $w$ on $\mathbb{R}^{d}$ such that $w(t)=\sigma(\|t\|)$, where $\sigma(r)$ is an increasing 
continuous concave function on $[0, \infty)$ with

$$
\begin{aligned}
& \sigma(0)=0 \\
& \int_{0}^{\infty} \frac{\sigma(r)}{1+r^{2}} d r<\infty, \\
& \sigma(r) \geq c+d \log (1+r) \quad \text { for some } c \in \mathbb{R}, d>0 .
\end{aligned}
$$

Consequently, $w \in \mathcal{M}$ implies that $w$ is subadditive, i.e., $w\left(t_{1}+t_{2}\right) \leq$ $w\left(t_{1}\right)+w\left(t_{2}\right)$ for all $t_{1}, t_{2} \in \mathbb{R}^{d}$, and thus we have for $\lambda>0$ that $m=e^{\lambda w}$ is submultiplicative, i.e., $m(0)=1$ and

$$
m\left(t_{1}+t_{2}\right) \leq m\left(t_{1}\right) m\left(t_{2}\right) \quad \forall t_{1}, t_{2} \in \mathbb{R}^{d} .
$$

A standard class of weight functions is given by

$$
m_{s}(t)=(1+\|t\|)^{s} \quad \text { for } s \in \mathbb{R} .
$$

For $s>0$, the function $m_{s}$ is submultiplicative, and we have $w_{s}=\log \left(m_{s}\right) \in$ $\mathcal{M}$.

Definition. For $w \in \mathcal{M}$, the space $\mathcal{S}_{w}\left(\mathbb{R}^{d}\right)$ of ultra-rapidly decaying test functions [28] is the Fréchet space of functions on $\mathbb{R}^{d}$ generated by the family of seminorms

$$
\mathcal{A}_{w}=\left\{\left\|e^{\lambda w} \mathrm{D}^{p} f\right\|_{\boldsymbol{L}^{\infty}},\left\|e^{\mu w} \mathrm{D}^{q} \widehat{f}\right\|_{\boldsymbol{L}^{\infty}}: \lambda, \mu>0, p, q \in \mathbb{N}_{0}^{d}\right\} .
$$

Its dual is the space $\mathcal{S}_{w}^{\prime}\left(\mathbb{R}^{d}\right)$ of tempered ultra-distributions. Here continuity of a linear functional $f$ means that there exist constants $C, \lambda, \mu>0$ and integers $M, N \geq 0$ such that for all $g \in \mathcal{S}_{w}\left(\mathbb{R}^{d}\right)$,

$$
|\langle f, g\rangle| \leq C\left(\sum_{\|p\|_{1} \leq M}\left\|e^{\lambda w} \mathrm{D}^{p} g\right\|_{\boldsymbol{L}^{\infty}}+\sum_{\|q\|_{1} \leq N}\left\|e^{\mu w} \mathrm{D}^{q} \widehat{g}\right\|_{\boldsymbol{L}^{\infty}}\right) .
$$

Obviously, if we choose $w(t)=\log (1+\|t\|)$, we obtain $\mathcal{S}_{w}\left(\mathbb{R}^{d}\right)=\mathcal{S}\left(\mathbb{R}^{d}\right)$, the Schwartz space [26], and consequently $\mathcal{S}_{w}^{\prime}\left(\mathbb{R}^{d}\right)=\mathcal{S}^{\prime}\left(\mathbb{R}^{d}\right)$, the space of tempered distributions.

Lemma 2.1. Given $w \in \mathcal{M}$, we have for $\lambda>0$ that

$$
\left\|e^{\lambda w}(f * g)\right\|_{\boldsymbol{L}^{\infty}} \leq C_{\lambda}\left\|e^{2 \lambda w} f\right\|_{\boldsymbol{L}^{\infty}}\left\|e^{2 \lambda w} g\right\|_{\boldsymbol{L}^{\infty}}
$$

for a constant $C_{\lambda}$ depending on $\lambda$ only. 
Proof. We show first that

$$
\int_{\mathbb{R}^{d}} e^{-2 \lambda w(t)} e^{-2 \lambda w(x-t)} d t \leq C_{\lambda} e^{-\lambda w(x)} .
$$

For $\|t-x\| \leq\|x\| / 2$, we have $\|t\| \geq\|x\| / 2$, so

$$
\begin{aligned}
\int_{\mathbb{R}^{d}} e^{-2 \lambda(w(t)+w(x-t))} d t \leq & \int_{\|t-x\| \leq \frac{\|x\|}{2}} e^{-2 \lambda w(x / 2)} e^{-2 \lambda w(t-x)} d t \\
& +\int_{\|t-x\| \geq \frac{\|x\|}{2}} e^{-2 \lambda w(t)} e^{-2 \lambda w(x / 2)} d t \\
\leq & e^{-2 \lambda w(x / 2)} 2 \int_{\mathbb{R}^{d}} e^{-\lambda w(t)} d t \\
\leq & e^{-\lambda w(x)} C_{\lambda}
\end{aligned}
$$

where in the last inequality, we made use of $w(x)=\sigma(\|x\|)$ with $\sigma$ concave and $\sigma(0)=0$, thus $\sigma(\|x / 2\|) \geq(\sigma(0)+\sigma(\|x\|)) / 2=\sigma(\|x\|) / 2$.

Now, we use

$$
|f(t)| \leq\left\|e^{2 \lambda w} f\right\|_{\boldsymbol{L}^{\infty}} e^{-2 \lambda w(t)} \quad \text { and } \quad|g(t)| \leq\left\|e^{2 \lambda w} g\right\|_{\boldsymbol{L}^{\infty}} e^{-2 \lambda w(t)},
$$

so

$$
\begin{aligned}
|(f * g)(x)| & \leq(|f| *|g|)(x) \\
& \leq\left\|e^{2 \lambda w} f\right\|_{\boldsymbol{L}^{\infty}}\left\|e^{2 \lambda w} g\right\|_{\boldsymbol{L}^{\infty}} \int_{\mathbb{R}^{d}} e^{-2 \lambda w(t)} e^{-2 \lambda w(x-t)} d t \\
& \leq\left\|e^{2 \lambda w} f\right\|_{\boldsymbol{L}^{\infty}}\left\|e^{2 \lambda w} g\right\|_{\boldsymbol{L}^{\infty}} C_{\lambda} e^{-\lambda w(x)}
\end{aligned}
$$

which implies the claim.

2.2. STFT on $\mathcal{S}_{w}\left(\mathbb{R}^{d}\right)$ and $\mathcal{S}_{w}^{\prime}\left(\mathbb{R}^{d}\right)$. For a fixed non-zero window $g \in \mathcal{S}_{w}\left(\mathbb{R}^{d}\right)$, the STFT is well-defined for $f \in \mathcal{S}_{w}^{\prime}\left(\mathbb{R}^{d}\right)$ and maps a tempered ultra-distribution on $\mathbb{R}^{d}$ to a function on $\mathbb{R}^{d} \times \widehat{\mathbb{R}}^{d}$.

Now we want to show how the STFT reflects properties of ultra-rapidly decaying test functions and of tempered ultra-distributions. First, we need an algebraic lemma for interchanging the operators $e^{\lambda w} D^{p}$ and $M_{\xi} T_{x}$. 
Lemma 2.2. For $g \in \mathcal{S}_{w}\left(\mathbb{R}^{d}\right)$, we have

$$
\begin{aligned}
\text { (i) } \quad e^{\lambda w} \mathrm{D}^{p}\left(\mathrm{M}_{\xi} \mathrm{T}_{x} g\right) & =\sum_{r \leq p}\left(\begin{array}{l}
p \\
r
\end{array}\right)(2 \pi i \xi)^{r} \mathrm{M}_{\xi} \mathrm{T}_{x}\left(e^{\lambda w(\cdot+x)} \mathrm{D}^{p-r} g\right), \\
\text { (ii) }\left\|e^{\lambda w} \mathrm{D}^{p}\left(\mathrm{M}_{\xi} \mathrm{T}_{x} g\right)\right\|_{\boldsymbol{L}^{\infty}} & \leq e^{\lambda w(x)} \sum_{r \leq p}\left(\begin{array}{l}
p \\
r
\end{array}\right)\left|(2 \pi i \xi)^{r}\right|\left\|e^{\lambda w} \mathrm{D}^{p-r} g\right\|_{\boldsymbol{L}^{\infty}} .
\end{aligned}
$$

Proof. (i) Obviously, we have

$$
\left(e^{\lambda w} \mathrm{~T}_{x} g\right)(t)=e^{\lambda w(t)} g(t-x)=\mathrm{T}_{x}\left(e^{\lambda w(t+x)} g(t)\right) .
$$

Furthermore, Leibniz's rule implies

$$
\begin{aligned}
\left(\mathrm{D}^{p} \mathrm{M}_{\xi} h\right)(t)=\mathrm{D}^{p}\left(e^{2 \pi i \xi t} h(t)\right) & =\sum_{r \leq p}\left(\begin{array}{l}
p \\
r
\end{array}\right)\left(\mathrm{D}^{r} e^{2 \pi i \xi t}\right)\left(D^{p-r} h(t)\right) \\
& =\left(\sum_{r \leq p}\left(\begin{array}{l}
p \\
r
\end{array}\right)(2 \pi i \xi)^{r} \mathrm{M}_{\xi} \mathrm{D}^{p-r} h\right)(t)
\end{aligned}
$$

Combining (2.3) and (2.4) yields

$$
\begin{aligned}
e^{\lambda w}\left(\mathrm{D}^{p} \mathrm{M}_{\xi}\left(\mathrm{T}_{x} g\right)\right) & =e^{\lambda w}\left(\sum_{r \leq p}\left(\begin{array}{l}
p \\
r
\end{array}\right)(2 \pi i \xi)^{r} \mathrm{M}_{\xi} \mathrm{D}^{p-r}\left(\mathrm{~T}_{x} g\right)\right) \\
& =\sum_{r \leq p}\left(\begin{array}{l}
p \\
r
\end{array}\right)(2 \pi i \xi)^{r} \mathrm{M}_{\xi}\left(e^{\lambda w} \mathrm{~T}_{x}\left(\mathrm{D}^{p-r} g\right)\right) \\
& =\sum_{r \leq p}\left(\begin{array}{l}
p \\
r
\end{array}\right)(2 \pi i \xi)^{r} \mathrm{M}_{\xi}\left(\mathrm{T}_{x} e^{\lambda w(\cdot+x)}\left(\mathrm{D}^{p-r} g\right)\right) .
\end{aligned}
$$

(ii) follows from (i) by using the isometry property $\left\|\mathrm{M}_{\xi} \mathrm{T}_{x} h\right\|_{\boldsymbol{L}^{\infty}}=$ $\|h\|_{L^{\infty}}$ and the submultiplicativity $e^{\lambda w(t+x)} \leq e^{\lambda w(t)} e^{\lambda w(x)}$.

Corollary 2.3. Time-frequency shifts act continuously on $\mathcal{S}_{w}$ and weak ${ }^{*}$-continuously on $\mathcal{S}_{w}^{\prime}$.

Proof. We have to show that for $g \in \mathcal{S}_{w}\left(\mathbb{R}^{d}\right)$,

$$
\lim _{\|x\|,\|\xi\| \rightarrow 0}\left\|e^{\lambda w(t)} \mathrm{D}^{p}\left(\mathrm{M}_{\xi} \mathrm{T}_{x} g-g\right)\right\|_{\boldsymbol{L}^{\infty}}=0
$$

and

$$
\lim _{\|x\|,\|\xi\| \rightarrow 0}\left\|e^{\mu w(\tau)} \mathrm{D}^{q}\left(\widehat{\mathrm{M}_{\xi} \mathrm{T}_{x}} g-\widehat{g}\right)\right\|_{\boldsymbol{L}_{\infty}}=0
$$


for all $\lambda, \mu>0, p, q \in \mathbb{N}_{0}^{d}$. According to Lemma 2.2, we have

$$
\begin{aligned}
\left\|e^{\lambda w(t)} \mathrm{D}^{p}\left(\mathrm{M}_{\xi} \mathrm{T}_{x} g-g\right)\right\|_{L^{\infty}} \leq & \left\|e^{\lambda w(t)}\left(\mathrm{M}_{\xi} \mathrm{T}_{x} \mathrm{D}^{p} g-\mathrm{D}^{p} g\right)\right\|_{L^{\infty}} \\
& +e^{\lambda w(x)} \sum_{\substack{r \leq p \\
r \neq 0}}\left(\begin{array}{l}
p \\
r
\end{array}\right)\left|(2 \pi i \xi)^{r}\right|\left\|e^{\lambda w(t)} \mathrm{D}^{p-r} g\right\|_{L^{\infty}} .
\end{aligned}
$$

The convergence of the first term, as $\|x\|,\|\xi\| \rightarrow 0$, is immediate for $g \in C^{\infty}$ with compact support. Since compactly supported functions are dense in $\mathcal{S}_{w}\left(\mathbb{R}^{d}\right)$, the convergence in the general case follows from a $3 \varepsilon$-argument. The terms in $\sum_{r}$ converge to 0 , because $r \neq 0$.

The convergence of $\left\|e^{\mu w(\tau)} \mathrm{D}^{q}\left(\widehat{\mathrm{M}_{\xi} \mathrm{T}_{x}} g-\widehat{g}\right)\right\|_{L^{\infty}}$ is treated similarly.

Consequently, for $f \in \mathcal{S}_{w}^{\prime}\left(\mathbb{R}^{d}\right)$, we have for all $g \in \mathcal{S}_{w}\left(\mathbb{R}^{d}\right)$ that

$$
\lim _{\|x\|,\|\xi\| \rightarrow 0}\left\langle\mathrm{M}_{\xi} \mathrm{T}_{x} f, g\right\rangle=\lim _{\|x\|,\|\xi\| \rightarrow 0}\left\langle f, \mathrm{~T}_{-x} \mathrm{M}_{-\xi} g\right\rangle=\langle f, g\rangle,
$$

which shows the weak*-continuity in $\mathcal{S}_{w}^{\prime}\left(\mathbb{R}^{d}\right)$.

This last statement implies that the STFT of a tempered ultradistribution is a continuous function on the time-frequency plane. We can say more about its growth, and we can also characterize elements of $\mathcal{S}_{w}\left(\mathbb{R}^{d}\right)$ by the decay properties of their STFT, as the following results show.

Theorem 2.4. Let $g \in \mathcal{S}_{w}\left(\mathbb{R}^{d}\right)$ and $f \in \mathcal{S}_{w}^{\prime}\left(\mathbb{R}^{d}\right)$. Then $\mathcal{V}_{g} f$ is continuous, and there are constants $C, \lambda, \mu>0$ such that

$$
\left|\left(\mathcal{V}_{g} f\right)(x, \xi)\right| \leq C e^{\lambda w(x)+\mu w(\xi)} \quad \forall x, \xi \in \mathbb{R}^{d} .
$$

Proof. The continuity of $f$ as a linear functional on $\mathcal{S}_{w}\left(\mathbb{R}^{d}\right)$ yields by $(2.2)$

$$
\begin{aligned}
\left|\left(\mathcal{V}_{g} f\right)(x, \xi)\right|= & \left|\left\langle f, \mathrm{M}_{\xi} \mathrm{T}_{x} g\right\rangle\right| \\
\leq & C\left(\sum_{\|p\|_{1} \leq M}\left\|e^{\lambda w} \mathrm{D}^{p}\left(\mathrm{M}_{\xi} \mathrm{T}_{x} g\right)\right\|_{\boldsymbol{L}^{\infty}}\right. \\
& \left.+\sum_{\|q\|_{1} \leq N}\left\|e^{\mu w} \mathrm{D}^{q}\left(e^{2 \pi i \xi x} \mathrm{M}_{-x} \mathrm{~T}_{\xi} \widehat{g}\right)\right\|_{\boldsymbol{L}^{\infty}}\right) .
\end{aligned}
$$


With the identity of Lemma 2.2 , we obtain

$$
\begin{aligned}
\left|\left(\mathcal{V}_{g} f\right)(x, \xi)\right| \leq & C\left(\sum_{\|p\|_{1} \leq M} e^{\lambda w(x)} \sum_{r \leq p}\left(\begin{array}{l}
p \\
r
\end{array}\right)\left|(2 \pi i \xi)^{r}\right|\left\|e^{\lambda w} \mathrm{D}^{p-r} g\right\|_{\boldsymbol{L}^{\infty}}\right. \\
& \left.+\sum_{\|q\|_{1} \leq N} e^{\mu w(\xi)} \sum_{s \leq q}\left(\begin{array}{l}
q \\
s
\end{array}\right)\left|(-2 \pi i x)^{s}\right|\left\|e^{\mu w} \mathrm{D}^{q-s} \widehat{g}\right\|_{\boldsymbol{L}^{\infty}}\right) .
\end{aligned}
$$

Since $g \in \mathcal{S}_{w}\left(\mathbb{R}^{d}\right)$, the right hand side can be written as $C\left(e^{\lambda w(x)} q\left(\left|\xi_{1}\right|, \ldots\right.\right.$, $\left.\left.\left|\xi_{d}\right|\right)+e^{\mu w(\xi)} p\left(\left|x_{1}\right|, \ldots,\left|x_{d}\right|\right)\right)$, where $p$ and $q$ are polynomials of degree $N$ and $M$ on $\mathbb{R}^{d}$. Consequently we obtain $\left|\mathcal{V}_{g} f(x, \xi)\right| \leq C^{\prime} e^{\lambda^{\prime} w(x)+\mu^{\prime} w(\xi)}$ for all $\lambda^{\prime}>\lambda, \mu^{\prime}>\mu$. The continuity of $\mathcal{V}_{g} f$ follows from Corollary 2.3.

Corollary 2.5. Let $g \in \mathcal{S}\left(\mathbb{R}^{d}\right)$ and $f \in \mathcal{S}^{\prime}\left(\mathbb{R}^{d}\right)$. Then $\mathcal{V}_{g} f$ is continuous, and there are integers $M, N \geq 0$ and a constant $C>0$ such that

$$
\left|\left(\mathcal{V}_{g} f\right)(x, \xi)\right| \leq C(1+\|x\|)^{M}(1+\|\xi\|)^{N} \quad \forall x, \xi \in \mathbb{R}^{d} .
$$

Proposition 2.6. Let $g \in \mathcal{S}_{w}\left(\mathbb{R}^{d}\right)$ be fixed. Assume that $F: \mathbb{R}^{2 d} \rightarrow \mathbb{C}$ has ultra-rapid decay, i.e., that for all $\lambda>0$, there is a constant $C_{\lambda}>0$ such that

$$
|F(x, \xi)| \leq C_{\lambda} e^{-\lambda(w(x)+w(\xi))}
$$

Then the integral

$$
f(t):=\iint_{\mathbb{R}^{2 d}} F(x, \xi)\left(\mathrm{M}_{\xi} \mathrm{T}_{x} g\right)(t) d x d \xi
$$

defines a function $f$ in $\mathcal{S}_{w}\left(\mathbb{R}^{d}\right)$.

Proof. The integral in (2.5) is absolutely convergent in $t$. Thus we may differentiate under the integral sign as long as the resulting integral is absolutely convergent, uniformly on compact sets. The latter is certainly true by virtue of the assumptions on $g$ and $F$. Thus we obtain with Lemma 2.2

$$
\begin{aligned}
\left(e^{\lambda w} \mathrm{D}^{p} f\right)(t) & =\iint F(x, \xi) e^{\lambda w(t)} \mathrm{D}^{p}\left(\mathrm{M}_{\xi} \mathrm{T}_{x} g\right)(t) d x d \xi \\
& =\sum_{r \leq p}\left(\begin{array}{l}
p \\
r
\end{array}\right) \iint F(x, \xi)(2 \pi i \xi)^{r} \mathrm{M}_{\xi} \mathrm{T}_{x}\left(e^{\lambda w(\cdot+x)} \mathrm{D}^{p-r} g\right)(t) d x d \xi
\end{aligned}
$$


SO

$$
\begin{aligned}
\left\|e^{\lambda w} \mathrm{D}^{p} f\right\|_{L^{\infty}} & \leq \sum_{r \leq p}\left(\begin{array}{l}
p \\
r
\end{array}\right) \iint|F(x, \xi)|\left|(2 \pi i \xi)^{r}\right| e^{\lambda w(x)}\left\|e^{\lambda w} \mathrm{D}^{p-r} g\right\|_{\boldsymbol{L}^{\infty}} d x d \xi \\
& \leq C \iint|F(x, \xi)| P(x, \xi) d x d \xi
\end{aligned}
$$

where $C=\max _{r \leq p}\left\|e^{\lambda w} \mathrm{D}^{p-r} g\right\|_{\boldsymbol{L}^{\infty}}$ and

$$
P(x, \xi)=\sum_{r \leq p}\left(\begin{array}{l}
p \\
r
\end{array}\right)\left|(2 \pi i \xi)^{r}\right| e^{\lambda w(x)}=e^{\lambda w(x)} \prod_{j=1}^{d}\left(1+\left|2 \pi \xi_{j}\right|\right)^{p_{j}} .
$$

Similarly, the identity

$$
\begin{aligned}
\left(e^{\mu w} \mathrm{D}^{q} \widehat{f}\right)(\tau)= & \iint F(x, \xi) e^{\mu w(\tau)} \mathrm{D}^{q}\left(e^{2 \pi i \xi x} \mathrm{M}_{-x} \mathrm{~T}_{\xi} \widehat{g}\right)(\tau) d x d \xi \\
= & \sum_{s \leq q}\left(\begin{array}{l}
q \\
s
\end{array}\right) \iint F(x, \xi)(-2 \pi i x)^{s} \\
& \cdot e^{2 \pi i \xi x} \mathrm{M}_{-x} \mathrm{~T}_{\xi}\left(e^{\mu w(\cdot+\xi)} \mathrm{D}^{q-s} \widehat{g}\right)(\tau) d x d \xi
\end{aligned}
$$

yields the estimate

$\left\|e^{\mu w} \mathrm{D}^{q} \widehat{f}\right\|_{\boldsymbol{L}^{\infty}} \leq D \iint|F(x, \xi)| Q(x, \xi) d x d \xi$,

where $D=\max _{s \leq q}\left\|e^{\mu w} \mathrm{D}^{q-s} \widehat{g}\right\|_{\boldsymbol{L}^{\infty}}$ and $Q(x, \xi)=e^{\mu w(\xi)} \prod_{j=1}^{d}(1+$ $\left.\left|2 \pi x_{j}\right|\right)^{q_{j}}$.

The assumption on $F$ implies $\left\|e^{\lambda w} \mathrm{D}^{p} f\right\|_{\boldsymbol{L}^{\infty}}<\infty$ and $\left\|e^{\mu w} \mathrm{D}^{q} \widehat{f}\right\|_{\boldsymbol{L}^{\infty}}<$ $\infty$ for all $\lambda, \mu>0$, and consequently $f \in \mathcal{S}_{w}\left(\mathbb{R}^{d}\right)$.

Theorem 2.7. Let $g \in \mathcal{S}_{w}\left(\mathbb{R}^{d}\right) \backslash\{0\}$ be fixed. Then for $f \in \mathcal{S}_{w}^{\prime}\left(\mathbb{R}^{d}\right)$, the following are equivalent:

(i) $f \in \mathcal{S}_{w}\left(\mathbb{R}^{d}\right)$.

(ii) For all $\lambda>0$, there is $C_{\lambda}>0$ such that

$$
\left|\left(\mathcal{V}_{g} f\right)(x, \xi)\right| \leq C_{\lambda} e^{-\lambda(w(x)+w(\xi))} \quad \forall(x, \xi) \in \mathbb{R}^{2 d} .
$$

(iii) $\mathcal{V}_{g} f \in \mathcal{S}_{w}\left(\mathbb{R}^{2 d}\right)$. 
Proof. (ii) $\Rightarrow$ (i). For the special case $F=\mathcal{V}_{g} f$ in (2.5), we obtain the inversion formula (Lemma 1.1.(ii)). Thus by Proposition 2.6, we have $f \in \mathcal{S}_{w}\left(\mathbb{R}^{d}\right)$.

(i) $\Rightarrow$ (ii). Let $\lambda>0$ and write $\widetilde{g}(t)=\overline{g(-t)}$. Then

$$
\begin{aligned}
e^{2 \lambda w(x)}\left|\left(\mathcal{V}_{g} f\right)(x, \xi)\right| & =e^{2 \lambda w(x)}\left|\int_{\mathbb{R}^{d}} f(t) \widetilde{g}(x-t) e^{-2 \pi i \xi t} d t\right| \\
& \leq e^{2 \lambda w(x)} \int_{\mathbb{R}^{d}}|f(t)||\widetilde{g}(x-t)| d t \\
& =e^{2 \lambda w(x)}(|f| *|\widetilde{g}|)(x) \\
& \leq\left\|e^{2 \lambda w}(|f| *|\widetilde{g}|)\right\|_{\boldsymbol{L}^{\infty}} \\
& \leq C_{2 \lambda}\left\|e^{4 \lambda w} f\right\|_{\boldsymbol{L}^{\infty}}\left\|e^{4 \lambda w} \widetilde{g}\right\|_{\boldsymbol{L}^{\infty}}=: C
\end{aligned}
$$

(by Lemma 2.1,)

which implies

$$
\left|\left(\mathcal{V}_{g} f\right)(x, \xi)\right| \leq C e^{-2 \lambda w(x)}
$$

Analogously, using Lemma 1.1(vi)

$$
\left|\left(\mathcal{V}_{g} f\right)(x, \xi)\right|=\left|\left(\mathcal{V}_{\widehat{g}} \widehat{f}\right)(\xi,-x)\right| \leq D e^{-2 \lambda w(\xi)},
$$

where $D=C_{2 \lambda}\left\|e^{4 \lambda w} \widehat{f}\right\|_{\boldsymbol{L}^{\infty}}\left\|e^{4 \lambda w} \widetilde{\widehat{g}}\right\|_{\boldsymbol{L}^{\infty}}$. The square root of the product yields

$$
\left|\left(\mathcal{V}_{g} f\right)(x, \xi)\right| \leq \sqrt{C D} e^{-\lambda(w(x)+w(\xi))} .
$$

Since this holds for all $\lambda>0$, the STFT $\mathcal{V}_{g} f$ decays ultra-rapidly as claimed.

(i) $\Rightarrow$ (iii). Given $f, g \in \mathcal{S}_{w}\left(\mathbb{R}^{d}\right)$, the function $F(x, t)=f(t) \overline{g(t-x)}$ is in $\mathcal{S}_{w}\left(\mathbb{R}^{2 d}\right)$. Since $\mathcal{S}_{w}$ is invariant under partial Fourier transformations, we obtain that $\mathcal{V}_{g} f(x, \xi)=\int F(x, t) e^{-2 \pi i \xi t} d t=\mathcal{F}_{t} F$ is also in $\mathcal{S}_{w}\left(\mathbb{R}^{2 d}\right)$.

(iii) $\Rightarrow$ (ii). Obvious.

Corollary 2.8. Let $g \in \mathcal{S}\left(\mathbb{R}^{d}\right) \backslash\{0\}$ be fixed. Then for $f \in \mathcal{S}^{\prime}\left(\mathbb{R}^{d}\right)$, the following are equivalent:

(i) $f \in \mathcal{S}\left(\mathbb{R}^{d}\right)$. 
(ii) For all $n \geq 0$, there is $C_{n}>0$ such that

$$
\left|\left(\mathcal{V}_{g} f\right)(x, \xi)\right| \leq C_{n}(1+\|x\|+\|\xi\|)^{-n} \quad \forall(x, \xi) \in \mathbb{R}^{2 d} .
$$

(iii) $\mathcal{V}_{g} f \in \mathcal{S}\left(\mathbb{R}^{2 d}\right)$.

Remark. (i) Corollary 2.8 can be considered folklore, it is implicitely given in [12], occurs in [17, 14] and also follows from an abstract result about the smoothness of square integrable representations of nilpotent Lie groups [9].

(ii) A more economic proof of Theorem 2.7 requires only the implications (i) $\Rightarrow$ (iii) $\Rightarrow$ (ii) $\Rightarrow$ (i). For the sole proof of the theorem, the step (i) $\Rightarrow$ (ii) is not necessary. Note, however, that we have proved the following much stronger result, which has already been obtained in [8] with completely different methods. For the special case of the Schwartz space $\mathcal{S}\left(\mathbb{R}^{d}\right)$, this was already obtained in $[6,24]$.

Corollary 2.9. The space $\mathcal{S}_{w}\left(\mathbb{R}^{d}\right)$ can also be defined as the Fréchet space of functions on $\mathbb{R}^{d}$ generated by the family of seminorms

$$
\widetilde{\mathcal{A}}_{w}=\left\{\left\|e^{\lambda w} f\right\|_{\boldsymbol{L}^{\infty}},\left\|e^{\mu w} \widehat{f}\right\|_{\boldsymbol{L}^{\infty}}: \lambda, \mu>0\right\} .
$$

Proof. Consider $\mathcal{S}_{w}\left(\mathbb{R}^{d}\right)$ and $\widetilde{\mathcal{S}_{w}}\left(\mathbb{R}^{d}\right)$ to be the Fréchet spaces of functions on $\mathbb{R}^{d}$ generated by the family of seminorms $\mathcal{A}_{w}$ (defined in (2.1)) and $\widetilde{\mathcal{A}}_{w}$, respectively. Since $\mathcal{A}_{w} \supseteq \widetilde{\mathcal{A}}_{w}$, we obviously have $\mathcal{S}_{w}\left(\mathbb{R}^{d}\right) \subseteq \widetilde{\mathcal{S}_{w}}\left(\mathbb{R}^{d}\right)$, and the embedding is continuous. On the other hand, in the proof of Theorem 2.7, (i) $\Rightarrow$ (ii), we only used the seminorms in $\widetilde{\mathcal{A}}_{w}$ to obtain that $f \in \mathcal{S}_{w}\left(\mathbb{R}^{d}\right)$. This shows that $\mathcal{S}_{w}\left(\mathbb{R}^{d}\right)=\widetilde{\mathcal{S}_{w}}\left(\mathbb{R}^{d}\right)$ as sets. So we know that the embedding $\mathcal{S}_{w}\left(\mathbb{R}^{d}\right) \rightarrow \widetilde{\mathcal{S}_{w}}\left(\mathbb{R}^{d}\right)$ is a continuous bijection, and thus by the open mapping theorem for Fréchet spaces it is a homeomorphism.

\section{Spaces of type $S$}

The spaces $\mathcal{S}_{w}\left(\mathbb{R}^{d}\right)$ were defined by the ultra-rapid decay of all derivatives $\mathrm{D}^{p} f$ and $\mathrm{D}^{p} \hat{f}$. An alternative strategy to construct useful spaces of test functions is to impose conditions on the growth of the norms $\left\|\mathrm{X}^{p} \mathrm{D}^{q} f\right\|_{\boldsymbol{L}^{\infty}\left(\mathbb{R}^{d}\right)}$ as a function of the order $p, q \in \mathbb{N}_{0}^{d}$, where $\mathrm{X}$ is the multiplication operator $\left(\mathrm{X}^{p} f\right)(x)=x^{p} f(x)$. This approach leads to the Gelfand-Shilov spaces and will be treated next.

Recall that we write $A^{p}=\left(A_{1}, \ldots, A_{d}\right)^{\left(p_{1}, \ldots, p_{d}\right)}=\prod_{i=1}^{d} A_{i}^{p_{i}}$ and $p^{p \alpha}=$ $\prod_{i=1}^{d} p_{i}^{p_{i} \alpha_{i}}$ for $A \in \mathbb{R}^{d}, p \in \mathbb{N}_{0}^{d}$ and $\alpha \geq 0$.

Definition. ([13], $\S$ IV.9) Let $\alpha, \beta \geq 0$. 
(a) We define $\boldsymbol{S}_{\alpha}$ to be the space of all functions $f \in \boldsymbol{C}^{\infty}\left(\mathbb{R}^{d}\right)$ with the property that there exist constants $A_{1}, \ldots, A_{d}, C_{q}>0$ such that

$$
\left\|\mathrm{X}^{p} \mathrm{D}^{q} f\right\|_{\boldsymbol{L}^{\infty\left(\mathbb{R}^{d}\right)}} \leq C_{q} A^{p} p^{p \alpha} \quad \forall p, q \in \mathbb{N}_{0}^{d} .
$$

(b) We define $\boldsymbol{S}^{\beta}$ to be the space of all functions $f \in \boldsymbol{C}^{\infty}\left(\mathbb{R}^{d}\right)$ with the property that there exist constants $B_{1}, \ldots, B_{d}, C_{p}>0$ such that

$$
\left\|\mathrm{X}^{p} \mathrm{D}^{q} f\right\|_{\boldsymbol{L}^{\infty}\left(\mathbb{R}^{d}\right)} \leq C_{p} B^{q} q^{q \beta} \quad \forall p, q \in \mathbb{N}_{0}^{d} .
$$

(c) We define $\boldsymbol{S}_{\alpha}^{\beta}$ to be the space of all functions $f \in \boldsymbol{C}^{\infty}\left(\mathbb{R}^{d}\right)$ with the property that there exist constants $A_{1}, \ldots, A_{d}, B_{1}, \ldots, B_{d}, C>0$ such that

$$
\left\|\mathrm{X}^{p} \mathrm{D}^{q} f\right\|_{\boldsymbol{L}^{\infty}\left(\mathbb{R}^{d}\right)} \leq C A^{p} p^{p \alpha} B^{q} q^{q \beta} \quad \forall p, q \in \mathbb{N}_{0}^{d} .
$$

The topology on the spaces of type $\boldsymbol{S}$ is defined by declaring which sequences are convergent.

Definition. A sequence $\left(f_{n}\right)_{n \in \mathbb{N}}$ in a space of type $\boldsymbol{S}$ converges to 0 , if the constants in (3.1)-(3.3) can be chosen uniformly in $n$, and for each $q \in \mathbb{N}_{0}^{d}$, we have $\mathrm{D}^{q} f_{n} \rightarrow 0$ uniformly on compact sets.

\section{Lemma 3.1.}

(i) ([13], § IV.9) For any $\alpha, \beta \geq 0$, the spaces $\boldsymbol{S}_{\alpha}$ and $\boldsymbol{S}^{\beta}$ are nontrivial. Also, $\boldsymbol{S}_{\alpha}^{\beta}$ is nontrivial, if and only if for each $i=$ $1, \ldots, d$, we have $\alpha_{i}+\beta_{i}>1$, or $\alpha_{i}+\beta_{i}=1$ and $\alpha_{i} \beta_{i}>0$. Furthermore, each nontrivial space of type $\boldsymbol{S}$ is rich in the sense that if $\varphi \in \boldsymbol{L}_{\text {loc }}^{1}\left(\mathbb{R}^{d}\right)$ satisfies $\int_{\mathbb{R}^{d}} f \varphi=0$ for all $f \in \boldsymbol{S}_{\alpha}$ (or $\left.\boldsymbol{S}^{\beta}\right)$, then $\varphi=0$.

(ii) ([13], $\S$ IV.9) $\widehat{\boldsymbol{S}_{\alpha}}=\boldsymbol{S}^{\alpha}, \widehat{\boldsymbol{S}^{\beta}}=\boldsymbol{S}_{\beta}, \widehat{\boldsymbol{S}_{\alpha}^{\beta}}=\boldsymbol{S}_{\beta}^{\alpha}$.

(iii) (Kashpirovskij's theorem [22]) $\boldsymbol{S}_{\alpha} \cap \boldsymbol{S}^{\beta}=\boldsymbol{S}_{\alpha}^{\beta}$.

(iv) ([13], § IV.4\&6) Each space of type $\boldsymbol{S}$ is invariant under the operators $\mathrm{X}, \mathrm{D}, \mathrm{T}_{x}$, and $\mathrm{M}_{\xi}$.

In view of Lemma 3.1 we will carry out the proofs as follows: (a) we will first prove each statement for the class $\boldsymbol{S}_{\alpha}$, (b) then the corresponding statement for $\boldsymbol{S}^{\beta}$ is obtained by taking Fourier transform, (c) finally, the statement for $\boldsymbol{S}_{\alpha}^{\beta}$ is a consequence of combining (a) and (b) with Kashpirovskij's theorem.

3.1. STFT on spaces of type $\boldsymbol{S}$. For a fixed window $g$ in a space of type $\boldsymbol{S}$, the STFT is well-defined for any $f$ in the corresponding dual space, and thus maps appropriate distributions on $\mathbb{R}^{d}$ to functions on $\mathbb{R}^{d} \times \widehat{\mathbb{R}}^{d}$. 
We want to show how the STFT reflects properties of such functions and distributions. First, we provide an algebraic lemma for interchanging the operators $\mathrm{X}^{p} \mathrm{D}^{q}$ and $\mathrm{M}_{\xi} \mathrm{T}_{x}$.

Lemma 3.2 ([17, L. 2.1]). For $g \in \mathcal{S}\left(\mathbb{R}^{d}\right)$, we have

$$
\mathrm{X}^{p} \mathrm{D}^{q}\left(\mathrm{M}_{\xi} \mathrm{T}_{x} g\right)=\sum_{r \leq p} \sum_{s \leq q}\left(\begin{array}{l}
p \\
r
\end{array}\right)\left(\begin{array}{l}
q \\
s
\end{array}\right) x^{r}(2 \pi i \xi)^{s} \mathrm{M}_{\xi} \mathrm{T}_{x}\left(\mathrm{X}^{p-r} \mathrm{D}^{q-s} g\right) .
$$

\section{Corollary 3.3.}

(i) Time-frequency shifts act continuously on all spaces of type $\boldsymbol{S}$, and weak ${ }^{*}$-continuously on their duals.

(ii) Let $g$ be an element of a space of type $\boldsymbol{S}$, and $f$ in the dual space. Then $\mathcal{V}_{g} f$ is continuous.

Proof. (i) We saw in Section 2 already that time-frequency shifts act continuously on $\mathcal{S}$. For any $f$ in a space of type $\boldsymbol{S}$, we have $f \in \mathcal{S}$, and thus

$$
\mathrm{D}^{q}\left(\mathrm{M}_{\xi} \mathrm{T}_{x} f-f\right) \rightarrow 0 \text { uniformly on compact sets, }
$$

for all $q \in \mathbb{N}_{0}^{d}$.

For $f \in \boldsymbol{S}_{\alpha}$, we have $\left\|\mathrm{X}^{p} \mathrm{D}^{q} f\right\|_{\boldsymbol{L}^{\infty}} \leq C_{q} A^{p} p^{p \alpha}$. This implies

$$
\begin{aligned}
\left\|\mathrm{X}^{p} \mathrm{D}^{q}\left(\mathrm{M}_{\xi} \mathrm{T}_{x} f\right)\right\|_{L^{\infty}} & \leq \sum_{r \leq p} \sum_{s \leq q}\left(\begin{array}{l}
p \\
r
\end{array}\right)\left(\begin{array}{l}
q \\
s
\end{array}\right)\left\|x^{r}(2 \pi i \xi)^{s} \mathrm{M}_{\xi} \mathrm{T}_{x}\left(\mathrm{X}^{p-r} \mathrm{D}^{q-s} f\right)\right\|_{\boldsymbol{L}^{\infty}} \\
& \leq \sum_{r \leq p} \sum_{s \leq q}\left(\begin{array}{l}
p \\
r
\end{array}\right)\left(\begin{array}{l}
q \\
s
\end{array}\right)|x|^{r}(2 \pi|\xi|)^{s}\left\|\mathrm{X}^{p-r} \mathrm{D}^{q-s} f\right\|_{\boldsymbol{L}^{\infty}} \\
& \leq \sum_{r \leq p} \sum_{s \leq q}\left(\begin{array}{l}
p \\
r
\end{array}\right)\left(\begin{array}{l}
q \\
s
\end{array}\right)|x|^{r}(2 \pi|\xi|)^{s} C_{q-s} A^{p-r}(p-r)^{(p-r) \alpha} \\
& \leq\left(\max _{s \leq q} C_{s}\right)(A+|x|)^{p} p^{p \alpha}(1+2 \pi|\xi|)^{q},
\end{aligned}
$$

where in a slight abuse of notation, we have written $A+|x|=$ $\left(A_{1}+\left|x_{1}\right|, \ldots, A_{d}+\left|x_{d}\right|\right)$ and $1+2 \pi|\xi|=\left(1+2 \pi\left|\xi_{1}\right|, \ldots, 1+2 \pi\left|\xi_{d}\right|\right)$. So we have for $|x|,|\xi| \leq 1$ that

$$
\left\|\mathrm{X}^{p} \mathrm{D}^{q}\left(\mathrm{M}_{\xi} \mathrm{T}_{x} f-f\right)\right\|_{L^{\infty}} \leq C_{q}^{\prime}(A+1)^{p} p^{p \alpha},
$$

and thus $\mathrm{M}_{\xi} \mathrm{T}_{x} f-f \rightarrow 0$ in $\boldsymbol{S}_{\alpha}$ as $(x, \xi) \rightarrow(0,0)$.

Consequently, for $f \in \boldsymbol{S}_{\alpha}^{\prime}$, we have for all $g \in \boldsymbol{S}_{\alpha}$ that

$$
\lim _{\|x\|,\|\xi\| \rightarrow 0}\left\langle\mathrm{M}_{\xi} \mathrm{T}_{x} f, g\right\rangle=\lim _{\|x\|,\|\xi\| \rightarrow 0}\left\langle f, \mathrm{~T}_{-x} \mathrm{M}_{-\xi} g\right\rangle=\langle f, g\rangle,
$$


which shows the weak*-continuity in $\boldsymbol{S}_{\alpha}^{\prime}$.

$\boldsymbol{S}^{\beta}$ and $\boldsymbol{S}_{\alpha}^{\beta}$ are treated analogously.

(ii) is an immediate consequence of (i).

For the next result, we will need the following estimate.

Lemma 3.4. Let $\alpha=\left(\alpha_{1}, \ldots, \alpha_{d}\right)>0$. Then for all $k=\left(k_{1}, \ldots, k_{d}\right) \geq 0$, there exist constants $C$ and $A=\left(A_{1}, \ldots, A_{d}\right)$ depending on $k$ and $\alpha$ such that

$$
(p+k)^{(p+k) \alpha} \leq C A^{p} p^{p \alpha} \quad \forall p \in \mathbb{N}_{0}^{d} .
$$

Proof. Without loss of generality we may assume that $d=1$. We write

$$
(p+k)^{(p+k) \alpha}=(p+k)^{k \alpha}\left(\frac{p+k}{p}\right)^{p \alpha} p^{p \alpha},
$$

and observe that

$$
(p+k)^{k \alpha} \leq e^{(p+k) k \alpha}=e^{k^{2} \alpha}\left(e^{k \alpha}\right)^{p},
$$

and

$$
\left(\frac{p+k}{p}\right)^{p \alpha}=\left(1+\frac{1}{p / k}\right)^{(p / k) k \alpha} \rightarrow e^{k \alpha} \quad(p \rightarrow \infty),
$$

so $\left(\frac{p+k}{p}\right)^{p \alpha}$ is bounded. The choice $C=e^{k^{2} \alpha} \sup _{p \in \mathbb{N}_{0}}\left(\frac{p+k}{p}\right)^{p \alpha}$ and $A=e^{k \alpha}$ now yields the claim.

The following result is analogous to Proposition 2.6 for $\mathcal{S}_{w}\left(\mathbb{R}^{d}\right)$. We write $\max \left\{\alpha, \alpha^{\prime}\right\}=\left(\max \left\{\alpha_{1}, \alpha_{1}^{\prime}\right\}, \ldots, \max \left\{\alpha_{d}, \alpha_{d}^{\prime}\right\}\right)$ for $\alpha, \alpha^{\prime} \in \mathbb{R}^{d}$.

Proposition 3.5. Let $\alpha, \alpha^{\prime}, \beta, \beta^{\prime} \geq 0$, and denote $\alpha^{\prime \prime}=\max \left\{\alpha, \alpha^{\prime}\right\}$, $\beta^{\prime \prime}=\max \left\{\beta, \beta^{\prime}\right\}$. Assume $F: \mathbb{R}^{d} \times \widehat{\mathbb{R}}^{d} \rightarrow \mathbb{C}$ and $h: \mathbb{R}^{d} \rightarrow \mathbb{C}$, and consider

$$
f(t)=\iint_{\mathbb{R}^{d} \times \widehat{\mathbb{R}}^{d}} F(x, \xi)\left(\mathrm{M}_{\xi} \mathrm{T}_{x} h\right)(t) d x d \xi .
$$

(a) If there are constants $A_{1}, \ldots, A_{d}, C_{q}>0$ such that

$$
\left\|x^{p} \xi^{q} F(x, \xi)\right\|_{\boldsymbol{L}^{\infty}\left(\mathbb{R}^{d} \times \widehat{\mathbb{R}}^{d}\right)} \leq C_{q} A^{p} p^{p \alpha} \quad \forall p, q \in \mathbb{N}_{0}^{d},
$$

and $h \in \boldsymbol{S}_{\alpha^{\prime}}$, then $f \in \boldsymbol{S}_{\alpha^{\prime \prime}}$.

(b) If there are constants $B_{1}, \ldots, B_{d}, C_{p}>0$ such that

$$
\left\|x^{p} \xi^{q} F(x, \xi)\right\|_{\boldsymbol{L}^{\infty}\left(\mathbb{R}^{\left.d \times \widehat{\mathbb{R}}^{d}\right)}\right.} \leq C_{p} B^{q} q^{q \beta} \quad \forall p, q \in \mathbb{N}_{0}^{d},
$$

and $h \in \boldsymbol{S}^{\beta^{\prime}}$, then $f \in \boldsymbol{S}^{\beta^{\prime \prime}}$.

(c) If there are constants $A_{1}, \ldots, A_{d}, B_{1}, \ldots, B_{d}, C>0$ such that

$$
\left\|x^{p} \xi^{q} F(x, \xi)\right\|_{\boldsymbol{L}^{\infty}\left(\mathbb{R}^{d} \times \widehat{\mathbb{R}}^{d}\right)} \leq C A^{p} p^{p \alpha} B^{q} q^{q \beta} \quad \forall p, q \in \mathbb{N}_{0}^{d},
$$


and $h \in \boldsymbol{S}_{\alpha^{\prime}}^{\beta^{\prime}}$, then $f \in \boldsymbol{S}_{\alpha^{\prime \prime}}^{\beta^{\prime \prime}}$.

Proof. (a) First, we show that the assumption on $F$ implies

$$
\iint_{\mathbb{R}^{d} \times \widehat{\mathbb{R}}^{d}}\left|x^{p} \xi^{q} F(x, \xi)\right| d x d \xi \leq C_{q} A^{p} p^{p \alpha} \quad \forall p, q \in \mathbb{N}_{0}^{d},
$$

with modified constants $C_{q}$ and $A$. Writing again $1+|x|=\left(1+\left|x_{1}\right|, \ldots\right.$, $\left.1+\left|x_{d}\right|\right)$ etc., we observe that

$$
\begin{aligned}
& \iint_{\mathbb{R}^{d} \times \widehat{\mathbb{R}}^{d}}\left|x^{p} \xi^{q} F(x, \xi)\right| d x d \xi \leq \iint_{\mathbb{R}^{d} \times \widehat{\mathbb{R}}^{d}}(1+|x|)^{p}(1+|\xi|)^{q}|F(x, \xi)| d x d \xi \\
& \leq\left\|(1+|x|)^{p+2}(1+|\xi|)^{q+2}|F(x, \xi)|\right\|_{\boldsymbol{L}^{\infty}\left(\mathbb{R}^{d} \times \widehat{\mathbb{R}}^{d}\right)} \\
& \cdot \iint_{\mathbb{R}^{d} \times \widehat{\mathbb{R}}^{d}} \prod_{i=1}^{d}\left(1+\left|x_{i}\right|\right)^{-2}\left(1+\left|\xi_{i}\right|\right)^{-2} d x d \xi \\
& \leq 2^{2 d} \sum_{r \leq p+2} \sum_{s \leq q+2}\left(\begin{array}{c}
p+2 \\
r
\end{array}\right)\left(\begin{array}{c}
q+2 \\
s
\end{array}\right)\left\|x^{r} \xi^{s} F(x, \xi)\right\|_{L^{\infty}\left(\mathbb{R}^{d} \times \widehat{\mathbb{R}}^{d}\right)} \\
& \leq 2^{2 d} \sum_{r \leq p+2} \sum_{s \leq q+2}\left(\begin{array}{c}
p+2 \\
r
\end{array}\right)\left(\begin{array}{c}
q+2 \\
s
\end{array}\right) C_{s} A^{r} r^{r \alpha}
\end{aligned}
$$

(now use $r^{r \alpha} \leq(p+2)^{(p+2) \alpha}:$ )

$$
\begin{aligned}
& \leq 2^{2 d}\left(\sum_{s \leq q+2}\left(\begin{array}{c}
q+2 \\
s
\end{array}\right) C_{s}\right)\left(\sum_{r \leq p+2}\left(\begin{array}{c}
p+2 \\
r
\end{array}\right) A^{r}\right)(p+2)^{(p+2) \alpha} \\
& \leq 2^{2 d}\left(\sum_{s \leq q+2}\left(\begin{array}{c}
q+2 \\
s
\end{array}\right) C_{s}\right)(1+A)^{p+2}(p+2)^{(p+2) \alpha} \\
& \quad \leq \widetilde{C}_{q} \widetilde{A}^{p} p^{p \alpha} \\
& \text { (Lemma 3.4) }
\end{aligned}
$$

as claimed.

By the assumption on $F$, the integral in (3.4) is absolutely convergent. Thus we may differentiate under the integral sign, as long as the resulting integral is absolutely convergent, uniformly on compact sets. But the latter is certainly true by virtue of the assumptions on $F$ and $h$, and we obtain 
with Lemma 3.2

$$
\begin{aligned}
\left(\mathrm{X}^{p} \mathrm{D}^{q} f\right)(t)= & \iint F(x, \xi) \mathrm{X}^{p} \mathrm{D}^{q}\left(\mathrm{M}_{\xi} \mathrm{T}_{x} g\right)(t) d x d \xi \\
= & \sum_{r \leq p} \sum_{s \leq q}\left(\begin{array}{l}
p \\
r
\end{array}\right)\left(\begin{array}{l}
q \\
s
\end{array}\right) \iint F(x, \xi) x^{r}(2 \pi i \xi)^{s} \\
& \cdot \mathrm{M}_{\xi} \mathrm{T}_{x}\left(\mathrm{X}^{p-r} \mathrm{D}^{q-s} h\right)(t) d x d \xi,
\end{aligned}
$$

so with $\left\|\mathrm{X}^{p} \mathrm{D}^{q} h\right\|_{\boldsymbol{L}^{\infty}} \leq C_{q}^{\prime} A^{p p} p^{p \alpha^{\prime}}$,

$$
\begin{aligned}
&\left\|\mathrm{X}^{p} \mathrm{D}^{q} f\right\|_{\boldsymbol{L}^{\infty}} \leq \sum_{r \leq p} \sum_{s \leq q}\left(\begin{array}{l}
p \\
r
\end{array}\right)\left(\begin{array}{l}
q \\
s
\end{array}\right) \iint|F(x, \xi)|\left|x^{r}\right|\left|(2 \pi i \xi)^{s}\right| \\
& \cdot\left\|\mathrm{X}^{p-r} \mathrm{D}^{q-s} h\right\|_{\boldsymbol{L}^{\infty}} d x d \xi \\
& \leq \sum_{r \leq p} \sum_{s \leq q}\left(\begin{array}{l}
p \\
r
\end{array}\right)\left(\begin{array}{l}
q \\
s
\end{array}\right)(2 \pi)^{s} C_{s} A^{r} r^{r \alpha} C_{q-s}^{\prime} A^{\prime p-r}(p-r)^{(p-r) \alpha^{\prime}}
\end{aligned}
$$

(now use $r^{r \alpha} \leq p^{r \alpha^{\prime \prime}}$ and $(p-r)^{(p-r) \alpha^{\prime}} \leq p^{(p-r) \alpha^{\prime \prime}}$ :)

$$
\begin{aligned}
& \leq\left(\sum_{s \leq q}\left(\begin{array}{c}
q \\
s
\end{array}\right)(2 \pi)^{s} C_{s} C_{q-s}^{\prime}\right)\left(\sum_{r \leq p}\left(\begin{array}{l}
p \\
r
\end{array}\right) A^{r} A^{\prime p-r}\right) p^{p \alpha^{\prime \prime}} \\
& =C_{q}^{\prime \prime}\left(A+A^{\prime}\right)^{p} p^{p \alpha^{\prime \prime}},
\end{aligned}
$$

so $f \in \boldsymbol{S}_{\alpha^{\prime \prime}}$ as claimed.

(b) The Fourier transformation applied to (3.4) yields

$$
\begin{aligned}
\widehat{f} & =\iint_{\mathbb{R}^{d} \times \widehat{\mathbb{R}}^{d}} F(x, \xi) \widehat{\mathrm{M}_{\xi} \mathrm{T}_{x}} h d x d \xi \\
& =\iint_{\mathbb{R}^{d} \times \widehat{\mathbb{R}}^{d}} F(x, \xi) e^{2 \pi i \xi x} \mathrm{M}_{-x} \mathrm{~T}_{\xi} \widehat{h} d x d \xi \\
& =\iint_{\mathbb{R}^{d} \times \widehat{\mathbb{R}}^{d}} F(-\eta, y) e^{-2 \pi i \eta y} \mathrm{M}_{\eta} \mathrm{T}_{y} \widehat{h} d y d \eta .
\end{aligned}
$$


Note that by the assumption on $F$, the function $\widetilde{F}(y, \eta)=e^{-2 \pi i \eta y} F(-\eta, y)$ satisfies

$$
\begin{aligned}
\left\|y^{p} \eta^{q} \widetilde{F}(y, \eta)\right\|_{\boldsymbol{L}^{\infty}\left(\mathbb{R}^{\left.d \times \widehat{\mathbb{R}}^{d}\right)}\right.} & =\left\|y^{p} \eta^{q} F(-\eta, y)\right\|_{\boldsymbol{L}^{\infty}\left(\mathbb{R}^{d} \times \widehat{\mathbb{R}}^{d}\right)} \\
& =\left\|(-x)^{q} \xi^{p} F(x, \xi)\right\|_{\boldsymbol{L}^{\infty}\left(\mathbb{R}^{d} \times \widehat{\mathbb{R}}^{d}\right)} \leq C_{q} B^{p} p^{p \beta},
\end{aligned}
$$

and that by Lemma 3.1.(ii), $\widehat{h} \in \boldsymbol{S}_{\beta^{\prime}}$. So we may apply (a) to $\widetilde{F}$ and $\widehat{h}$, which yields $\widehat{f} \in \boldsymbol{S}_{\beta^{\prime \prime}}$ or, again by Lemma 3.1.(ii), $f \in \boldsymbol{S}^{\beta^{\prime \prime}}$ as claimed.

(c) Immediate consequence from (a), (b), and Lemma 3.1.(iii).

Corollary 3.6. Let $\alpha, \beta \geq 0$, and $f, g \in \mathcal{S}\left(\mathbb{R}^{d}\right) \backslash\{0\}$.

(a) If there are constants $A_{1}, \ldots, A_{d}, C_{q}>0$ such that

$$
\left\|x^{p} \xi^{q}\left(\mathcal{V}_{g} f\right)(x, \xi)\right\|_{\boldsymbol{L}^{\infty}\left(\mathbb{R}^{d} \times \widehat{\mathbb{R}}^{d}\right)} \leq C_{q} A^{p} p^{p \alpha} \quad \forall p, q \in \mathbb{N}_{0}^{d},
$$

then $f, g \in \boldsymbol{S}_{\alpha}$.

(b) If there are constants $B_{1}, \ldots, B_{d}, C_{p}>0$ such that

$$
\left\|x^{p} \xi^{q}\left(\mathcal{V}_{g} f\right)(x, \xi)\right\|_{\boldsymbol{L}^{\infty}\left(\mathbb{R}^{d} \times \widehat{\mathbb{R}}^{d}\right)} \leq C_{p} B^{q} q^{q \beta} \quad \forall p, q \in \mathbb{N}_{0}^{d},
$$

then $f, g \in \boldsymbol{S}^{\beta}$.

(c) If there are constants $A_{1}, \ldots, A_{d}, B_{1}, \ldots, B_{d}, C>0$ such that

$$
\left\|x^{p} \xi^{q}\left(\mathcal{V}_{g} f\right)(x, \xi)\right\|_{\boldsymbol{L}^{\infty}\left(\mathbb{R}^{d} \times \widehat{\mathbb{R}}^{d}\right)} \leq C A^{p} p^{p \alpha} B^{q} q^{q \beta} \quad \forall p, q \in \mathbb{N}_{0}^{d},
$$

then $f, g \in \boldsymbol{S}_{\alpha}^{\beta}$.

Proof. Apply Proposition 3.5 with $F=\mathcal{V}_{g} f$ and appropriate $h$. Note that the existence of appropriate candidates for $h$ is guaranteed by Lemma 3.1.(i).

Corollary 3.7 (Uncertainty principle). Let $f, g \in \mathcal{S}\left(\mathbb{R}^{d}\right)$, and assume that for some $\alpha, \beta \geq 0$, there are constants $A_{1}, \ldots, A_{d}, B_{1}, \ldots, B_{d}, C>0$ such that

$$
\left\|x^{p} \xi^{q}\left(\mathcal{V}_{g} f\right)(x, \xi)\right\|_{\boldsymbol{L}^{\infty}\left(\mathbb{R}^{d} \times \widehat{\mathbb{R}}^{d}\right)} \leq C A^{p} p^{p \alpha} B^{q} q^{q \beta} \quad \forall p, q \in \mathbb{N}_{0}^{d} .
$$

If $\alpha_{i}+\beta_{i}<1$ or $\alpha_{i}+\beta_{i}=1$ and $\alpha_{i} \beta_{i}=0$ for some pair $\left(\alpha_{i}, \beta_{i}\right)$, i.e., if $\boldsymbol{S}_{\alpha}^{\beta}=\{0\}$, then $\mathcal{V}_{g} f \equiv 0$ and thus either $f \equiv 0$ or $g \equiv 0$.

A converse of Corollary 3.6 is contained in the next theorem.

Theorem 3.8. Let $\alpha, \alpha^{\prime}, \beta, \beta^{\prime} \geq 0$, and denote $\alpha^{\prime \prime}=\max \left\{\alpha, \alpha^{\prime}\right\}$, $\beta^{\prime \prime}=\max \left\{\beta, \beta^{\prime}\right\}$. 
(a) If $f$ and $g$ satisfy

$$
\begin{aligned}
& \left\|\mathrm{X}^{p} f\right\|_{\boldsymbol{L}^{\infty}\left(\mathbb{R}^{d}\right)} \leq C_{0} A^{p} p^{p \alpha} \quad \text { and } \quad\left\|\mathrm{X}^{q} \widehat{f}\right\|_{\boldsymbol{L}^{\infty}\left(\widehat{\mathbb{R}}^{d)}\right.}<\infty \quad \forall p, q \in \mathbb{N}_{0}^{d}, \\
& \left\|\mathrm{X}^{p} g\right\|_{\boldsymbol{L}^{\infty}\left(\mathbb{R}^{d}\right)} \leq C_{0}^{\prime} A^{\prime p} p^{p \alpha^{\prime}} \quad \text { and } \quad\left\|\mathrm{X}^{q} \widehat{g}\right\|_{\boldsymbol{L}^{\infty}\left(\widehat{\mathbb{R}}^{d}\right)}<\infty \quad \forall p, q \in \mathbb{N}_{0}^{d},
\end{aligned}
$$

(in particular, if $f \in \boldsymbol{S}_{\alpha}$ and $g \in \boldsymbol{S}_{\alpha^{\prime}}$ ),

then there exist constants $A_{1}^{\prime \prime}, \ldots, A_{d}^{\prime \prime}, C_{q}^{\prime \prime}>0$ such that

$$
\left\|x^{p} \xi^{q}\left(\mathcal{V}_{g} f\right)(x, \xi)\right\|_{\boldsymbol{L}^{\infty}\left(\mathbb{R}^{d} \times \widehat{\mathbb{R}}^{d}\right)} \leq C_{q}^{\prime \prime} A^{\prime \prime} p^{p \alpha^{\prime \prime}} \quad \forall p, q \in \mathbb{N}_{0}^{d} .
$$

(b) If $f$ and $g$ satisfy

$$
\begin{array}{llll}
\left\|\mathrm{X}^{p} f\right\|_{\boldsymbol{L}^{\infty}\left(\mathbb{R}^{d}\right)}<\infty & \text { and } & \left\|\mathrm{X}^{q} \widehat{f}\right\|_{\boldsymbol{L}^{\infty}\left(\widehat{\mathbb{R}}^{d}\right)} \leq C_{0} B_{q} q^{q \beta} & \forall p, q \in \mathbb{N}_{0}^{d}, \\
\left\|\mathrm{X}^{p} g\right\|_{\boldsymbol{L}^{\infty}\left(\mathbb{R}^{d}\right)}<\infty & \text { and } & \left\|\mathrm{X}^{q} \widehat{g}\right\|_{\boldsymbol{L}^{\infty}\left(\widehat{\mathbb{R}}^{d}\right)} \leq C_{0}^{\prime} B_{q}^{\prime} q^{q \beta^{\prime}} & \forall p, q \in \mathbb{N}_{0}^{d},
\end{array}
$$

(in particular, if $f \in \boldsymbol{S}^{\beta}$ and $g \in \boldsymbol{S}^{\beta^{\prime}}$ ),

then there exist constants $B_{1}^{\prime \prime}, \ldots, B_{d}^{\prime \prime}, C_{p}^{\prime \prime}>0$ such that

$$
\left\|x^{p} \xi^{q}\left(\mathcal{V}_{g} f\right)(x, \xi)\right\|_{\boldsymbol{L}^{\infty}\left(\mathbb{R}^{\left.d \times \widehat{\mathbb{R}}^{d}\right)}\right.} \leq C_{p}^{\prime \prime} B^{\prime \prime q} q^{q \beta^{\prime \prime}} \quad \forall p, q \in \mathbb{N}_{0}^{d} .
$$

(c) If $f$ and $g$ satisfy

$$
\begin{aligned}
& \left\|\mathrm{X}^{p} f\right\|_{\boldsymbol{L}^{\infty}\left(\mathbb{R}^{d}\right)} \leq C_{0} A^{p} p^{p \alpha} \quad \text { and }\left\|\mathrm{X}^{q} \widehat{f}\right\|_{L^{\infty}\left(\widehat{\mathbb{R}}^{d}\right)} \leq C_{0} B_{q} q^{q \beta} \quad \forall p, q \in \mathbb{N}_{0}^{d}, \\
& \left\|\mathrm{X}^{p} g\right\|_{\boldsymbol{L}^{\infty}\left(\mathbb{R}^{d}\right)} \leq C_{0}^{\prime} A^{\prime p} p^{p \alpha^{\prime}} \quad \text { and }\left\|\mathrm{X}^{q} \widehat{g}\right\|_{\boldsymbol{L}^{\infty}\left(\widehat{\mathbb{R}}^{d}\right)} \leq C_{0}^{\prime} B_{q}^{\prime} q^{q \beta^{\prime}} \quad \forall p, q \in \mathbb{N}_{0}^{d},
\end{aligned}
$$

(in particular, if $f \in \boldsymbol{S}_{\alpha}^{\beta}$ and $g \in \boldsymbol{S}_{\alpha^{\prime}}^{\beta^{\prime}}$ ),

then there exist constants $A_{1}^{\prime \prime}, \ldots, A_{d}^{\prime \prime}, B_{1}^{\prime \prime}, \ldots, B_{d}^{\prime \prime}, C^{\prime \prime}>0$ such that

$$
\left\|x^{p} \xi^{q}\left(\mathcal{V}_{g} f\right)(x, \xi)\right\|_{L^{\infty}\left(\mathbb{R}^{d} \times \widehat{\mathbb{R}}^{d}\right)} \leq C^{\prime \prime} A^{\prime \prime p} p^{p \alpha^{\prime \prime}} B^{\prime \prime q} q^{q \beta^{\prime \prime}} \quad \forall p, q \in \mathbb{N}_{0}^{d} .
$$

Proof. (a) We leave it to the reader to show (in complete analogy to the beginning of the proof of Proposition 3.5) that the assumption on $f$ implies

$$
\int_{\mathbb{R}^{d}}\left|t^{p} f(t)\right| d t \leq C_{0} A^{p} p^{p \alpha} \quad \forall p \in \mathbb{N}_{0}^{d},
$$

with modified constants $C_{0}$ and $A$.

This observation yields the estimate

$$
\left\|x^{p}\left(\mathcal{V}_{g} f\right)(x, \xi)\right\|_{\boldsymbol{L}^{\infty}} \leq \sup _{x \in \mathbb{R}^{d}}\left|x^{p}\right| \int_{\mathbb{R}^{d}}|f(t)||g(t-x)| d t
$$




$$
\begin{aligned}
& \leq \int_{\mathbb{R}^{d}}|f(t)| \sup _{x \in \mathbb{R}^{d}}\left|x^{p} g(t-x)\right| d t \\
& =\int_{\mathbb{R}^{d}}|f(t)|\left\|(t-x)^{p} g(x)\right\|_{\boldsymbol{L}^{\infty}} d t \\
& \leq \int_{\mathbb{R}^{d}}|f(t)| \sum_{r \leq p}\left(\begin{array}{l}
p \\
r
\end{array}\right)\left|t^{r}\right|\left\|(-x)^{p-r} g(x)\right\|_{\boldsymbol{L}^{\infty}} d t \\
& \leq \sum_{r \leq p}\left(\begin{array}{l}
p \\
r
\end{array}\right) \int_{\mathbb{R}^{d}}\left|t^{r} f(t)\right| d t C_{0}^{\prime} A^{\prime p-r}(p-r)^{(p-r) \alpha^{\prime}} \\
& \leq \sum_{r \leq p}\left(\begin{array}{l}
p \\
r
\end{array}\right) C_{0} A^{r} r^{r \alpha} C_{0}^{\prime} A^{\prime p-r}(p-r)^{(p-r) \alpha^{\prime}}
\end{aligned}
$$

(now use $r^{r \alpha} \leq p^{r \alpha^{\prime \prime}}$ and $(p-r)^{(p-r) \alpha^{\prime}} \leq p^{(p-r) \alpha^{\prime \prime}}$ :)

$$
\begin{aligned}
& \leq C_{0} C_{0}^{\prime} \sum_{r \leq p}\left(\begin{array}{l}
p \\
r
\end{array}\right) A^{r} A^{\prime p-r} p^{p \alpha^{\prime \prime}} \\
& =C_{0}^{\prime \prime}\left(A+A^{\prime}\right)^{p} p^{p \alpha^{\prime \prime}}
\end{aligned}
$$

Since the hypothesis on $f$ and $g$ imply that $f, g \in \mathcal{S}$, Corollary 2.8 shows that $\mathcal{V}_{g} f \in \mathcal{S}$ and consequently we have

$$
\left\|\xi^{q} \mathcal{V}_{g} f\right\|_{\boldsymbol{L}^{\infty}}=: \widetilde{C}_{q}^{\prime \prime}<\infty
$$

From this we obtain

$$
\begin{aligned}
\left\|x^{p} \xi^{q}\left(\mathcal{V}_{g} f\right)(x, \xi)\right\|_{\boldsymbol{L}^{\infty}} & =\left\|x^{2 p} \xi^{2 q}\left(\mathcal{V}_{g} f\right)^{2}\right\|_{\boldsymbol{L}^{\infty}}^{1 / 2} \\
& \leq\left\|x^{2 p} \mathcal{V}_{g} f\right\|_{\boldsymbol{L}^{\infty}}^{1 / 2}\left\|\xi^{2 q} \mathcal{V}_{g} f\right\|_{\boldsymbol{L}^{\infty}}^{1 / 2} \\
& \leq\left(C_{0}^{\prime \prime}\left(A+A^{\prime}\right)^{2 p}(2 p)^{2 p \alpha^{\prime \prime}}\right)^{1 / 2}{\widetilde{C_{2 q}}}_{2{ }^{\prime \prime}}^{1 / 2} \\
& =\sqrt{C_{0}^{\prime \prime} \widetilde{C}_{2 q}^{\prime \prime}}\left(\left(A+A^{\prime}\right) 2^{\alpha^{\prime \prime}}\right)^{p} p^{p \alpha^{\prime \prime}}
\end{aligned}
$$

(b) By Lemma 1.1.(ii), we may employ (a) to obtain

$$
\begin{aligned}
\left\|x^{p} \xi^{q}\left(\mathcal{V}_{g} f\right)(x, \xi)\right\|_{\boldsymbol{L}^{\infty}} & =\left\|\xi^{q} x^{p}\left(\mathcal{V}_{\widehat{g}} \widehat{f}\right)(\xi,-x)\right\|_{\boldsymbol{L}^{\infty}} \\
& \leq C_{p}^{\prime \prime} B^{\prime \prime q} q^{q \beta^{\prime \prime}} .
\end{aligned}
$$


(c) Now we may make use of both (a) and (b), which yields

$$
\begin{aligned}
\left\|x^{p} \xi^{q}\left(\mathcal{V}_{g} f\right)(x, \xi)\right\|_{\boldsymbol{L}^{\infty}} & =\left\|x^{2 p} \xi^{2 q}\left(\mathcal{V}_{g} f\right)^{2}\right\|_{\boldsymbol{L}^{\infty}}^{1 / 2} \\
& \leq\left\|x^{2 p} \mathcal{V}_{g} f\right\|_{\boldsymbol{L}^{\infty}}^{1 / 2}\left\|\xi^{2 q} \mathcal{V}_{g} f\right\|_{\boldsymbol{L}^{\infty}}^{1 / 2} \\
& \leq\left(C_{0}^{\prime \prime} A^{\prime \prime 2 p}(2 p)^{2 p \alpha^{\prime \prime}}\right)^{1 / 2}\left(\widetilde{C}_{0}^{\prime \prime} B^{\prime \prime 2 q}(2 q)^{2 q \beta^{\prime \prime}}\right)^{1 / 2} \\
& =\sqrt{C_{0}^{\prime \prime} \widetilde{C}_{0}^{\prime \prime}}\left(A^{\prime \prime} 2^{\alpha^{\prime \prime}}\right)^{p} p^{p \alpha^{\prime \prime}}\left(B^{\prime \prime} 2^{\beta^{\prime \prime}}\right)^{q} q^{q \beta^{\prime \prime}}
\end{aligned}
$$

Remark. In the proofs of Proposition 3.5.(b) and (c), we were able to apply (a) by making use of Lemma 3.1.(ii) and (iii). Note, though, that the proof of Proposition 3.5.(a) can be modified easily to prove (b) and (c) directly.

With this modification, Proposition 3.5 and Theorem 3.8 yield a new, fairly elegant proof of Kashpirovskij's theorem (Lemma 3.1.(iii)), namely, via the following characterizations of the spaces of type $\boldsymbol{S}$.

Corollary 3.9. Let $\alpha, \beta \geq 0$.

(a) $\boldsymbol{S}_{\alpha}$ is the space of all functions $f \in C^{\infty}\left(\mathbb{R}^{d}\right)$ with the property that there exist constants $A_{1}, \ldots, A_{d}, C_{0}>0$ such that

$$
\left\|\mathrm{X}^{p} f\right\|_{\boldsymbol{L}^{\infty}\left(\mathbb{R}^{d}\right)} \leq C_{0} A^{p} p^{p \alpha} \quad \text { and } \quad\left\|\mathrm{X}^{q} \widehat{f}\right\|_{\boldsymbol{L}^{\infty}\left(\widehat{\mathbb{R}}^{d}\right)}<\infty \quad \forall p, q \in \mathbb{N}_{0}^{d}
$$

(b) $\boldsymbol{S}^{\beta}$ is the space of all functions $f \in \boldsymbol{C}^{\infty}\left(\mathbb{R}^{d}\right)$ with the property that there exist constants $B_{1}, \ldots, B_{d}, C_{0}>0$ such that

$$
\left\|\mathrm{X}^{p} f\right\|_{\boldsymbol{L}^{\infty}\left(\mathbb{R}^{d}\right)}<\infty \quad \text { and } \quad\left\|\mathrm{X}^{q} \hat{f}\right\|_{\boldsymbol{L}^{\infty}\left(\widehat{\mathbb{R}}^{d}\right)} \leq C_{p} B^{q} q^{q \beta} \quad \forall p, q \in \mathbb{N}_{0}^{d} .
$$

(c) $\boldsymbol{S}_{\alpha}^{\beta}$ is the space of all functions $f \in \boldsymbol{C}^{\infty}\left(\mathbb{R}^{d}\right)$ with the property that there exist constants $A_{1}, \ldots, A_{d}, B_{1}, \ldots, B_{d}, C>0$ such that

$$
\left\|\mathrm{X}^{p} f\right\|_{\boldsymbol{L}^{\infty}\left(\mathbb{R}^{d}\right)} \leq C A^{p} p^{p \alpha} \text { and }\left\|\mathrm{X}^{q} \widehat{f}\right\|_{\boldsymbol{L}^{\infty}\left(\widehat{\mathbb{R}}^{d}\right)} \leq C B^{q} q^{q \beta} \forall p, q \in \mathbb{N}_{0}^{d} .
$$

A somewhat more general form of part (c) of the above can already be found in [7].

This characterization yields a much stronger result on $\mathcal{V}_{g} f$.

Corollary 3.10. Let $\alpha, \alpha^{\prime}, \beta, \beta^{\prime} \geq 0$, and denote $\alpha^{\prime \prime}=\max \left\{\alpha, \alpha^{\prime}\right\}$, $\beta^{\prime \prime}=\max \left\{\beta, \beta^{\prime}\right\}$. If $f \in \boldsymbol{S}_{\alpha}^{\beta}$ and $g \in \boldsymbol{S}_{\alpha^{\prime}}^{\bar{\beta}^{\prime}}$, then $\mathcal{V}_{g} f \in \boldsymbol{S}_{\left(\alpha^{\prime \prime}, \beta^{\prime \prime}\right)}^{\left(\beta^{\prime}, \alpha\right)}$. 
Proof. By Theorem 3.8.(c), there exist constants $A_{1}^{\prime \prime}, \ldots, A_{d}^{\prime \prime}$, $B_{1}^{\prime \prime}, \ldots, B_{d}^{\prime \prime}, C^{\prime \prime}>0$ such that

$$
\left\|x^{p} \xi^{q}\left(\mathcal{V}_{g} f\right)(x, \xi)\right\|_{L^{\infty}\left(\mathbb{R}^{d} \times \widehat{\mathbb{R}}^{d}\right)} \leq C^{\prime \prime} A^{\prime \prime p} p^{p \alpha^{\prime \prime}} B^{\prime \prime q} q^{q \beta^{\prime \prime}} \quad \forall p, q \in \mathbb{N}_{0}^{d} .
$$

On the other hand, since $\left(\widehat{\mathcal{V}_{g} f}\right)(\eta, y)=e^{2 \pi i \eta y} \overline{\widehat{g}(\eta)} f(-y)(c f .$, Lemma 1.1.(iii)) with $f \in \boldsymbol{S}_{\alpha}$ and $\widehat{g} \in \boldsymbol{S}_{\beta^{\prime}}$, we have

$$
\begin{aligned}
\left\|\eta^{p} y^{q}\left(\widehat{\mathcal{V}_{g} f}\right)(\eta, y)\right\|_{\boldsymbol{L}^{\infty}\left(\widehat{\mathbb{R}}^{d} \times \mathbb{R}^{d}\right)} & =\left\|\eta^{p} \widehat{g}(\eta)\right\|_{\boldsymbol{L}^{\infty}\left(\widehat{\mathbb{R}}^{d}\right)}\left\|y^{q} f(y)\right\|_{\boldsymbol{L}^{\infty}\left(\mathbb{R}^{d}\right)} \\
& \leq C^{\prime} B^{\prime p} p^{p \beta^{\prime}} C A^{q} q^{q \alpha}
\end{aligned}
$$

Now we apply Corollary 3.9.(c), which yields the claim.

For the case $\alpha=\alpha^{\prime}$ and $\beta=\beta^{\prime}$, we obtain a characterization of $\boldsymbol{S}_{\alpha}^{\beta}$ in terms of the STFT in analogy to Theorem 2.7.

Corollary 3.11. Let $\alpha, \beta \geq 0$, and $g \in \boldsymbol{S}_{\alpha}^{\beta} \backslash\{0\}$. Then for $f \in\left(\boldsymbol{S}_{\alpha}^{\beta}\right)^{\prime}$, the following are equivalent:

(i) $f \in \boldsymbol{S}_{\alpha}^{\beta}$.

(ii) There exist constants $A_{1}, \ldots, A_{d}, B_{1}, \ldots, B_{d}, C>0$ such that

$$
\left\|x^{p} \xi^{q}\left(\mathcal{V}_{g} f\right)(x, \xi)\right\|_{\boldsymbol{L}^{\infty}\left(\mathbb{R}^{d} \times \widehat{\mathbb{R}}^{d}\right)} \leq C A^{p} p^{p \alpha} B^{q} q^{q \beta} \quad \forall p, q \in \mathbb{N}_{0}^{d} .
$$

(iii) $\mathcal{V}_{g} f \in \boldsymbol{S}_{(\alpha, \beta)}^{(\beta, \alpha)}\left(\mathbb{R}^{2 d}\right)$.

3.2. Different characterizations of the spaces of type $S$. Spaces of type $\boldsymbol{S}$ possess an alternative characterization that resembles more the definition of the classes $\mathcal{S}_{w}\left(\mathbb{R}^{d}\right)$. Let us write $\lambda \cdot|\xi|^{1 / \alpha}=\sum_{i=1}^{d} \lambda_{i}\left|\xi_{i}\right|^{1 / \alpha_{i}}$. Then $\boldsymbol{S}_{\alpha}^{\beta}$ consists of all functions $f \in \boldsymbol{C}^{\infty}\left(\mathbb{R}^{d}\right)$ with the property that there exist constants $\lambda_{1}, \ldots, \lambda_{d}, \mu_{1}, \ldots, \mu_{d}>0$ such that

$$
\left\|e^{\lambda \cdot|x|^{1 / \alpha}} \mathrm{D}^{q} f\right\|_{\boldsymbol{L}^{\infty}\left(\mathbb{R}^{d}\right)}<\infty \quad \text { and } \quad\left\|e^{\mu \cdot|\xi|^{1 / \beta}} \mathrm{D}^{p} \widehat{f}\right\|_{\boldsymbol{L}^{\infty}\left(\widehat{\mathbb{R}}^{d}\right)}<\infty \forall p, q \in \mathbb{N}_{0}^{d}
$$

While (3.5) is similar to Definition given by (2.1), the ultra-rapid decay is required only for a single pair $(\lambda, \mu)$ instead of all $\lambda, \mu \geq 0$; this might explain the considerable difference in the technical treatment of $\mathcal{S}_{w}\left(\mathbb{R}^{d}\right)$ and $\boldsymbol{S}_{\alpha}^{\beta}$. Similar characterizations hold for $\boldsymbol{S}_{\alpha}$ and $\boldsymbol{S}^{\beta}$. See [13] for this aspect of the spaces of type $\boldsymbol{S}$.

Proceeding as in the previous section, it is then possible to show the following characterization of $\boldsymbol{S}_{\alpha}^{\beta}$ (and of $\boldsymbol{S}_{\alpha}$ and $\boldsymbol{S}^{\beta}$ ).

Proposition 3.12. Let $\alpha, \beta>0$, and $g \in \boldsymbol{S}_{\alpha}^{\beta} \backslash\{0\}$. Then for $f \in\left(\boldsymbol{S}_{\alpha}^{\beta}\right)^{\prime}$, the following are equivalent: 
(i) $f \in \boldsymbol{S}_{\alpha}^{\beta}$.

(ii) There exist constants $\lambda_{1}, \ldots, \lambda_{d}, \mu_{1}, \ldots, \mu_{d}>0$ such that

$$
\left\|e^{\lambda \cdot|x|^{1 / \alpha}+\mu \cdot|\xi|^{1 / \beta}}\left(\mathcal{V}_{g} f\right)(x, \xi)\right\|_{\boldsymbol{L}^{\infty}\left(\mathbb{R}^{d} \times \widehat{\mathbb{R}}^{d}\right)}<\infty .
$$

(iii) There exist constants $\lambda_{1}, \ldots, \lambda_{d}, \mu_{1}, \ldots, \mu_{d}>0$ such that

$$
\left\|e^{\lambda \cdot|x|^{1 / \alpha}} f\right\|_{\boldsymbol{L}^{\infty}\left(\mathbb{R}^{d}\right)}<\infty \quad \text { and }\left\|e^{\mu \cdot|\xi|^{1 / \beta}} \hat{f}\right\|_{\boldsymbol{L}^{\infty}\left(\widehat{\mathbb{R}}^{d}\right)}<\infty .
$$

Remark. The equivalence of (i) and (ii) is essentially proved in [5] (using the Wigner distribution instead of the STFT). Also, in [21], the Wigner distribution has been used to characterize spaces of Björck and of GelfandShilov type.

\section{Modulation Spaces}

So far we have investigated the joint time-frequency behavior of test functions and distributions by means of the short-time Fourier transform. We now reverse the point of view and consider spaces that are defined by the decay properties of the short-time Fourier transform. This class of spaces is known as modulation spaces and is well understood, see [14, Ch. 11-13]. We may thus use and apply results from the theory of modulation spaces to obtain new results about spaces of test functions. In this sense, the theory of modulation spaces sheds a new light on the theory of test functions and distributions. We recall the definition of a modulation space.

Definition. Let $\phi(t)=e^{-\pi\|t\|^{2}}$ be the Gaussian and $m$ a submultiplicative weight function on the time-frequency plane. Then we define $\boldsymbol{M}_{m}^{p}\left(\mathbb{R}^{d}\right)$ to be the space

$$
\boldsymbol{M}_{m}^{p}\left(\mathbb{R}^{d}\right)=\left\{f \in \mathcal{S}^{\prime}\left(\mathbb{R}^{d}\right): \mathcal{V}_{\phi} f \in \boldsymbol{L}_{m}^{p}\left(\mathbb{R}^{d} \times \widehat{\mathbb{R}}^{d}\right)\right\}
$$

with norm

$$
\|f\|_{M_{m}^{p}\left(\mathbb{R}^{d}\right)}=\left\|\mathcal{V}_{\phi} f\right\|_{\boldsymbol{L}_{m}^{p}\left(\mathbb{R}^{d} \times \widehat{\mathbb{R}}^{d}\right)}=\left(\int_{\mathbb{R}^{d} \times \widehat{\mathbb{R}}^{d}}\left|\left(\mathcal{V}_{\phi} f\right)(z)\right|^{p} m(z)^{p} d z\right)^{1 / p} .
$$

Remark. (i) For $1 \leq p \leq 2, \boldsymbol{M}_{m}^{p}$ is a subspace of $\boldsymbol{L}^{2}\left(\mathbb{R}^{d}\right)$, because $\boldsymbol{M}_{m}^{p} \subseteq \boldsymbol{M}^{p} \subseteq \boldsymbol{L}^{2}\left(\mathbb{R}^{d}\right)$ by [14, Thm. 12.2.2].

For other types of weights, so-called moderate weight functions, $\boldsymbol{M}_{m}^{p}$ is not necessarily a subspace of $\boldsymbol{L}^{2}$ or even of $\mathcal{S}^{\prime}$. Also, (4.1) is not the most 
general definition of modulation spaces: usually one uses mixed $\boldsymbol{L}^{p, q_{-n o r m s}}$ imposed on $\mathcal{V}_{g} f$; see [14] for a detailed discussion and full generality.

(ii) Note that $\boldsymbol{M}_{m}^{p}$ is always non-trivial. Since $m$ is submultiplicative, it grows at most exponentially, i.e., $|m(z)| \leq C e^{A\|z\|}$ for some $A, C>0$. Therefore, $\boldsymbol{M}_{m}^{p}$ contains at least the Gaussian $\phi$, since $\left|\left(\mathcal{V}_{\phi} \phi\right)(x, \xi)\right|=$ $e^{-\pi\left(x^{2}+\xi^{2}\right) / 2}$ [14, L. 1.5.2]. Since $M_{m}^{p}$ is invariant under time-frequency shifts, it contains all finite linear combinations of the time-frequency shifts $\mathrm{M}_{\xi} \mathrm{T}_{x} \phi$. A slightly different argument shows that $\boldsymbol{M}_{m}^{p}$ also contains all finite linear combinations of Hermite functions.

(iii) We are mostly interested in spaces that are invariant under the Fourier transform. In view of Lemma 1.1.(ii), this requires the additional property

$$
m(x, \xi) \leq C m(\xi,-x) \quad \text { for }(x, \xi) \in \mathbb{R}^{d} \times \widehat{\mathbb{R}}^{d} .
$$

If $m$ satisfies this condition, then the Fourier transform is an isomorphism on $\boldsymbol{M}_{m}^{p}$.

(iv) The definition of $\boldsymbol{M}_{m}^{p}$ is "fairly" independent of the choice of the particular window. More precisely, for every $g \in \boldsymbol{M}_{m}^{1} \backslash\{0\}$, there exist constants $A, B>0$ such that

$$
A\left\|\mathcal{V}_{\phi} f\right\|_{\boldsymbol{L}_{m}^{p}} \leq\left\|\mathcal{V}_{g} f\right\|_{\boldsymbol{L}_{m}^{p}} \leq B\left\|\mathcal{V}_{\phi} f\right\|_{\boldsymbol{L}_{m}^{p}},
$$

in other words, for every $g \in \boldsymbol{M}_{m}^{1} \backslash\{0\}$, we have that $\left\|\mathcal{V}_{g} f\right\|_{\boldsymbol{L}_{m}^{p}}$ is an equivalent norm on $\boldsymbol{M}_{m}^{p}$ [14, Thm. 11.3.7].

The concept of modulation spaces leads to an elegant description of spaces of test functions and distributions within time-frequency analysis.

\section{Proposition 4.1.}

(i) Let $m_{s}(z)=(1+\|z\|)^{s}$. Then $\mathcal{S}=\bigcap_{s>0} \boldsymbol{M}_{m_{s}}^{p}$ for all $p \in[1, \infty]$.

(ii) Let $w \in \mathcal{M}$ and set $m_{\lambda}(x, \xi)=e^{\lambda(w(x)+w(\xi))}$. Then $\mathcal{S}_{w}=$ $\bigcap_{\lambda \geq 0} \boldsymbol{M}_{m_{\lambda}}^{p}$ for all $p \in[1, \infty]$.

(iii) Let $\alpha, \beta, \lambda>0$ and set $m_{\lambda}(x, \xi)=e^{\lambda\left(|x|^{1 / \alpha}+|\xi|^{1 / \beta}\right)}$. Then $\boldsymbol{S}_{\alpha}^{\beta}=\bigcup_{\lambda>0} \boldsymbol{M}_{m_{\lambda}}^{p}$ for all $p \in[1, \infty]$.

Proof. For $p=\infty$, assertions (ii) and (iii) are reformulations of Theorem 2.7 and Proposition 3.12, respectively. For $p \in[1, \infty[$, the statements follow from the embeddings

$$
\boldsymbol{M}_{m_{\lambda+\varepsilon}}^{\infty} \subseteq \boldsymbol{M}_{m_{\lambda}}^{1} \subseteq \boldsymbol{M}_{m_{\lambda}}^{\infty} \text { for some } \varepsilon>0,
$$

where $\varepsilon$ depends on $w \in \mathcal{M}$ in (ii).

Statement (i) was already observed in [14, Prop. 11.3.1] and is a special case of (ii). 
It is worth noting that while for $\alpha, \beta \geq 1$, the weight functions $m_{\lambda}(x, \xi)=e^{\lambda\left(|x|^{1 / \alpha}+|\xi|^{1 / \beta}\right)}$ are submultiplicative, and thus $\boldsymbol{M}_{m_{\lambda}}^{p} \neq\{0\}$ by the remarks above, this is not true in general. In particular, if $\alpha_{i}+\beta_{i}<1$ for some $i$, then $\boldsymbol{M}_{m_{\lambda}}^{p}=\{0\}$ for all $\lambda>0$, and thus $\boldsymbol{S}_{\alpha}^{\beta}=\{0\}$ [3] (compare Lemma 3.1.(i)).

These representations of spaces of test functions allow us to use methods of time-frequency analysis for the investigation of $\mathcal{S}_{w}\left(\mathbb{R}^{d}\right)$ and $\boldsymbol{S}_{\alpha}^{\beta}$. As an example how to apply known results about modulation spaces, we provide a characterization of test functions by means of Gabor expansions.

By a Gabor expansion, we understand a series expansion of the form

$$
f=\sum_{k, l \in \mathbb{Z}^{d}}\left\langle f, \mathrm{M}_{\beta n} \mathrm{~T}_{\alpha k} g\right\rangle \mathrm{M}_{\beta n} \mathrm{~T}_{\alpha k} \gamma
$$

for some fixed pair of windows $(g, \gamma)$. The construction of Gabor expansions valid in $\boldsymbol{L}^{2}\left(\mathbb{R}^{d}\right)$ is well understood and we may use the textbook results $[10,14]$ without much ado. Assume that $\mathcal{G}(g, \alpha, \beta)$ is a frame for $\boldsymbol{L}^{2}\left(\mathbb{R}^{d}\right)$, meaning that the Gabor frame operator defined by $S f=$ $\sum_{k, l \in \mathbb{Z}^{d}}\left\langle f, \mathrm{M}_{\beta n} \mathrm{~T}_{\alpha k} g\right\rangle \mathrm{M}_{\beta n} \mathrm{~T}_{\alpha k} g$ is invertible on $\boldsymbol{L}^{2}\left(\mathbb{R}^{d}\right)$. Since

$$
f=S^{-1} S f=\sum_{k, l \in \mathbb{Z}^{d}}\left\langle f, \mathrm{M}_{\beta n} \mathrm{~T}_{\alpha k} g\right\rangle \mathrm{M}_{\beta n} \mathrm{~T}_{\alpha k} S^{-1} g,
$$

we can choose $\gamma=S^{-1} g \in \boldsymbol{L}^{2}\left(\mathbb{R}^{d}\right)$, see, e.g., [10] or [14]. A much deeper theorem of Janssen [19] states that if $S$ in invertible on $\boldsymbol{L}^{2}\left(\mathbb{R}^{d}\right)$ and if $g \in \mathcal{S}$, then also $\gamma=S^{-1} g \in \mathcal{S}$.

A similar statement can be derived for ultra test functions.

Theorem 4.2. Assume that $g \in \mathcal{S}_{w}$ and that the Gabor frame operator $S$ is invertible on $\boldsymbol{L}^{2}\left(\mathbb{R}^{d}\right)$. Then $\gamma=S^{-1} g \in \mathcal{S}_{w}$.

Proof. The statement follows by a combination of the main result in [15] and Prop. 4.1(ii). Set $m_{\lambda}(x, \xi)=e^{\lambda(w(x)+w(\xi))}$ as above. If $g \in \boldsymbol{M}_{m_{\lambda}}^{1}$ and $S$ is invertible on $\boldsymbol{L}^{2}\left(\mathbb{R}^{d}\right)$, then by [15, Thm. 4.2] $S$ is also invertible on $\boldsymbol{M}_{m_{\lambda}}^{1}$, and thus $\gamma=S^{-1} g \in M_{m_{\lambda}}^{1}$. Consequently, if $g \in \mathcal{S}_{w}=\bigcap_{\lambda \geq 0} \boldsymbol{M}_{m_{\lambda}}^{1}$, then also $\gamma \in \mathcal{S}_{w}$ by Prop. 4.1.(ii).

Using these facts, we obtain the following characterization of ultra-test functions in terms of their coefficients of Gabor expansions.

Theorem 4.3. Assume that $S$ is invertible on $\boldsymbol{L}^{2}\left(\mathbb{R}^{d}\right)$ and $g \in \mathcal{S}_{w}$ with dual window $\gamma \in \mathcal{S}_{w}$. Then (4.3) holds for $f \in \mathcal{S}_{w}$ with convergence in $\mathcal{S}_{w}$. Furthermore, $f \in \mathcal{S}_{w}$ if and only if $f$ has a Gabor expansion with 
coefficients $c_{k l}=\left\langle f, \mathrm{M}_{\beta n} \mathrm{~T}_{\alpha k} \gamma\right\rangle$ satisfying $\left|c_{k l}\right| \leq C_{\lambda} e^{-\lambda(w(\alpha k)+w(\beta l))}$ for all $\lambda \geq 0$.

Proof. Let $m_{\lambda}(x, \xi)=e^{\lambda(w(x)+w(\xi))}$, and apply [14, Cor. 12.2.6] to $\boldsymbol{M}_{m_{\lambda}}^{1}$ for each $\lambda \geq 0$.

If $w(t)=\log (1+\|t\|)$, then this yields a characterization of the Schwartz class $\mathcal{S}$ which was already observed in [17]. For $w(t)=\|t\|^{c}$, one obtains a characterization of ultra-test functions (see also [25]). For Wilson bases in place of Gabor frames, similar results have been obtained in $[11,16,25]$.

Finally, we check when a modulation space contains functions with compact support, more precisely, we ask when $\boldsymbol{M}_{m}^{1}$ contains functions of arbitrarily small support. As is to be expected, this question brings in the logarithmic integral and quasianalyticity.

Theorem 4.4. Assume that $m(x, \xi) \leq C m(\xi,-x)$ and set $m_{2}(\xi)=$ $m(0, \xi)$. Then the following are equivalent.

(i) $M_{m}^{1}$ contains functions of arbitrarily small support.

(ii) $\mathcal{F} \boldsymbol{L}_{m_{2}}^{1}$ contains functions of arbitrarily small support.

(iii) $m$ satisfies the logarithmic integral condition

$$
\int_{\|z\| \geq 1} \frac{\log m(z)}{\|z\|^{2 d+1}} d z<\infty .
$$

(iv) $m$ satisfies

$$
\int_{\|\xi\| \geq 1} \frac{\log m(0, \xi)}{\|\xi\|^{d+1}} d \xi<\infty .
$$

Proof. (ii) $\Longleftrightarrow$ (iv) is classical, see [4, p. 412] and [23] for details.

(ii) $\Longrightarrow$ (i). Assume that supp $f \subseteq[-A, A]^{d}$ and that $\int_{\widehat{\mathbb{R}}^{d}}|\widehat{f}(\xi)| m_{2}(\xi) d \xi<$ $\infty$. Then using Lemma 1.1 we obtain

$$
\left(\widehat{f} * \mathrm{M}_{-x} \widehat{f}^{*}\right)(\xi)=\left\langle\widehat{f}, \mathrm{~T}_{\xi} \mathrm{M}_{-x} \widehat{f}\right\rangle=\left\langle f, \mathrm{M}_{\xi} \mathrm{T}_{x} f\right\rangle=0
$$


for $\|x\|>2 A$. Therefore,

$$
\begin{aligned}
\iint_{\mathbb{R}^{d} \times \widehat{\mathbb{R}}^{d}}\left|\left(\mathcal{V}_{f} f\right)(x, \xi)\right| m(x, \xi) d x d \xi & \leq \int_{\|x\| \leq 2 A} \int_{\widehat{\mathbb{R}}^{d}}\left|\left(\widehat{f} * \mathrm{M}_{-x} \widehat{f}^{*}\right)(\xi)\right| m(0, \xi) d \xi \\
& \leq \int_{\|x\| \leq 2 A}\left\|\widehat{f} * \mathrm{M}_{-x} \widehat{f}^{*}\right\|_{\boldsymbol{L}_{m_{2}}^{1}} m(x, 0) d x \\
& \leq \int_{\|x\| \leq 2 A}\|\widehat{f}\|_{\boldsymbol{L}_{m_{2}}^{1}}\left\|\mathrm{M}_{-x} \widehat{f}\right\|_{\boldsymbol{L}_{m_{2}}^{1}} m(x, 0) d x \\
& \leq C\|\widehat{f}\|_{\boldsymbol{L}_{m_{2}}^{1}}^{2}<\infty .
\end{aligned}
$$

Since $\mathcal{V}_{f} f \in \boldsymbol{L}_{m}^{1}\left(\mathbb{R}^{d} \times \widehat{\mathbb{R}}^{d}\right)$ implies that $\mathcal{V}_{\phi} f \in \boldsymbol{L}_{m}^{1}\left(\mathbb{R}^{d} \times \widehat{\mathbb{R}}^{d}\right)$ by $[14$, Lemma 12.1.1], we have $f \in \boldsymbol{M}_{m}^{1}$. Consequently every compactly supported function in $\mathcal{F} \boldsymbol{L}_{m_{2}}^{1}$ is also in $\boldsymbol{M}_{m}^{1}$. The implication (ii) $\Longrightarrow$ (i) follows.

(i) $\Longrightarrow$ (iii). Next assume that for every $\varepsilon>0$, there exists $f$ with $\operatorname{supp} f \subseteq[-\varepsilon, \varepsilon]^{d}$ and $f \in \boldsymbol{M}_{m}^{1}$, i.e., $\mathcal{V}_{\phi} f \in \boldsymbol{L}_{m}^{1}\left(\mathbb{R}^{d} \times \widehat{\mathbb{R}}^{d}\right)$ where $\phi(t)=e^{-\pi t^{2}}$. Since under the assumptions made, $\boldsymbol{M}_{m}^{1}$ is invariant under the Fourier transform, we also have $\widehat{f} \in \boldsymbol{M}_{m}^{1}$. Invoking [14, Lemma 12.1.1], we derive that $\mathcal{V}_{\widehat{f}} f \in \boldsymbol{L}_{m}^{1}\left(\mathbb{R}^{d} \times \widehat{\mathbb{R}}^{d}\right)$. By Lemma 1.1.(iii), we find that

$$
\left(\widehat{\mathcal{V}_{\widehat{f}} f}\right)(\eta, y)=e^{-2 \pi i y \cdot \eta} f(-y) \widehat{\widehat{f}}(\eta)=e^{-2 \pi i y \cdot \eta} f(-y) f(-\eta),
$$

and thus supp $\widehat{\mathcal{V}_{\widehat{f}} f} \subseteq[-\varepsilon, \varepsilon]^{2 d}$. Consequently, $\mathcal{F} \boldsymbol{L}_{m}^{1}\left(\mathbb{R}^{d} \times \widehat{\mathbb{R}}^{d}\right)$ contains functions with arbitrarily small support, and by the theory of the logarithmic integral ([4, p. 412] and [23]), $m$ satisfies condition (4.4).

(iii) $\Longrightarrow$ (ii). If (4.4) is satisfied, then for every $\varepsilon>0$, there exists a function $F$ on $\mathbb{R}^{2 d}$ such that $\operatorname{supp} F \subseteq[-\varepsilon, \varepsilon]^{2 d}$ and $\int_{\widehat{\mathbb{R}}^{2 d}}|\widehat{F}(\zeta)| m(\zeta) d \zeta<\infty$. Fubini's theorem then implies that

$$
\int_{\widehat{\mathbb{R}}^{d}}|\widehat{F}(\omega, \xi)| m(0, \xi) d \xi \leq m(-\omega, 0) \int_{\widehat{\mathbb{R}}^{d}}|\widehat{F}(\omega, \xi)| m(\omega, \xi) d \xi<\infty
$$

for (almost) all $\omega \in \widehat{\mathbb{R}}^{d}$. For such an $\omega$, define the function $f$ on $\mathbb{R}^{d}$ by

$$
f(x)=\int_{\mathbb{R}^{d}} F(w, x) e^{-2 \pi i \omega \cdot w} d w .
$$


Then supp $f \subseteq[-\varepsilon, \varepsilon]^{d}$ and

$$
\widehat{f}(\xi)=\int_{\mathbb{R}^{d}} \int_{\mathbb{R}^{d}} F(w, x) e^{-2 \pi i \omega \cdot w} d w e^{-2 \pi i \xi \cdot x} d x=\widehat{F}(\omega, \xi) .
$$

Now (4.6) implies that $\int_{\widehat{\mathbb{R}}^{d}}|\widehat{f}(\xi)| m_{2}(\xi) d \xi<\infty$, in other words, $\mathcal{F} \boldsymbol{L}_{m_{2}}^{1}$ contains functions of arbitrarily small support.

In some applications, even stronger decay conditions on the short-time Fourier transform are considered, see, e.g., the superexponential decay conditions in the design of windows for Gabor frames [2]. In this case the weight function $v$ is no longer assumed to be submultiplicative and may thus grow faster than exponentially, e.g., $v(z)=e^{a\|z\|^{c}}$ for some $c>1$. It still makes sense to consider the modulation spaces $\boldsymbol{M}_{m}^{p}$, but they seem to lose all their basic properties. For instance, $\boldsymbol{M}_{m}^{p}$ may not be invariant unter time-frequency shifts, and the definition of $\boldsymbol{M}_{m}^{p}$ may depend on the choice of the window. Moreover, the version of Hardy's theorem for the STFT imposes a limit on the growth of $m$. If $m(z) \geq C e^{a\|z\|^{2}}$ for some $a>\pi / 2$, then the corresponding $\boldsymbol{M}_{m}^{1}$ contains only the trivial function $f \equiv 0$ [17].

\section{References}

[1] G. Björck, Linear partial differential operators and generalized distributions, Ark. Mat., 6 (1966), 351-407.

[2] H. Bölcskei and A. J. E. M. Janssen, Gabor frames, unimodularity, and window decay, J. Fourier Anal. Appl., 6 (2000), 255-276.

[3] A. Bonami, B. Demange and P. Jaming, Hermite functions and uncertainty principles for the Fourier and the windowed Fourier transforms, Rev. Mat. Iberoamericana, 19 (2003), 23-55.

[4] L. Carleson, P. Malliavin, J. Neuberger and J. Wermer, The collected works of Arne Beurling, Vol. 2: Harmonic analysis, Birkhäuser, Boston, 1989.

[5] J. Cho, A characterization of Gelfand-Shilov space based on Wigner distribution, Commun. Korean Math. Soc., 14 (1999), 761-767.

[6] J. Chung, S.-Y. Chung and D. Kim, Une caractérisation de l'espace $\mathcal{S}$ de Schwartz, C. R. Acad. Sci. Paris Sér. I Math., 316 (1993), 23-25.

[7] J. Chung, S.-Y. Chung and D. Kim, Characterization of the GelfandShilov spaces via Fourier transforms, Proc. Amer. Math. Soc., 124 (1996), 2101-2108. 
[8] S.-Y. Chung, D. Kim and S. Lee, Characterization for Beurling-Björck space and Schwartz space, Proc. Amer. Math. Soc., 125 (1997), 32293234 .

[9] L. Corwin, Matrix coefficients of nilpotent Lie groups, Lie group representations III, Proc. Spec. Year, College Park, MD, Lecture Notes in Math., 1077 (1984) 1-11.

[10] I. Daubechies, Ten lectures on wavelets, CBMS-NSF Series in Applied Mathematics, 61, SIAM, Philadelphia, 1992.

[11] H. G. Feichtinger, K. Gröchenig and D. Walnut, Wilson bases and modulation spaces, Math. Nachr., 155 (1992), 7-17.

[12] G. B. Folland, Harmonic Analysis on Phase Space, Ann. of Math. Stud., 121 (1989), Princeton Univ. Press, Princeton.

[13] I. M. Gelfand and G. E. Schilow, Verallgemeinerte Funktionen (Distributionen) II: Lineare Topologische Räume, Räume von Grundfunktionen und verallgemeinerten Funktionen, Hochschulbücher für Mathematik, 48, VEB Deutscher Verlag der Wissenschaften, Berlin, 1969.

[14] K. Gröchenig, Foundations of Time-Frequency Analysis, Birkhäuser, Boston, 2001.

[15] K. Gröchenig and M. Leinert, Wiener's lemma for twisted convolution and Gabor frames, preprint, 2001.

[16] K. Gröchenig and D. Walnut, A Riesz basis for Bargmann-Fock space related to sampling and interpolation, Ark. Mat., 30 (1992), 283-295.

[17] K. Gröchenig and G. Zimmermann, Hardy's theorem and the shorttime Fourier transform of Schwartz functions, J. London Math. Soc., 63 (2001), 205-214.

[18] L. Hörmander, The analysis of linear partial differential operators, I: Distribution theory and Fourier analysis, Springer, Berlin, 1990.

[19] A. J. E. M. Janssen, Duality and biorthogonality for Weyl-Heisenberg frames, J. Fourier Anal. Appl., 1 (1995) (4), 403-436.

[20] A. J. E. M. Janssen and S. J. L. van Eijndhoven, Spaces of type $W$, growth of Hermite coefficients, Wigner distribution, and Bargmann transform, J. Math. Anal. Appl., 152 (1990), 368-390.

[21] A. Kamiński, D. Perišić and S. Pilipović, On various integral transformations of tempered distributions, Demonstratio Math., 33 (2000), 641-655.

[22] A. I. Kashpirovskij, Equality of the spaces $\boldsymbol{S}_{\alpha}^{\beta}$ and $\boldsymbol{S}_{\alpha} \cap \boldsymbol{S}^{\beta}$, Funct. Anal. Appl., 14 (1980), 129. 
[23] P. Koosis, The logarithmic integral I, Cambridge Stud. Adv. Math., 12, Cambridge University Press, Cambridge, 1998.

[24] M. S. Osborne, On the Schwartz-Bruhat space and the Paley-Wiener theorem for locally compact abelian groups, J. Funct. Anal., 19 (1976), 40-49.

[25] S. Pilipović and N. Teofanov, Wilson bases and ultramodulation spaces, Math. Nachr., 242 (2002), 179-196.

[26] L. Schwartz, Théorie des distributions, Nouvelle édition, Publications de l'Institut de Mathématique de l'Université de Strasbourg, Hermann, Paris, 1966.

[27] N. Teofanov, A note on ultrapolynomials and the Wigner distribution, Novi Sad J. Math., 30 (2000), 165-175.

[28] H. Triebel, Theory of Function Spaces, Monographs Math., 78, Birkhäuser, Basel, 1983.

Department of Mathematics U-9

University of Connecticut

Storrs, CT 06269-3009

USA

(E-mail : groch@math.uconn.edu)

Institut für Angewandte Mathematik und Statistik

Universität Hohenheim

70593 Stuttgart

Germany

(E-mail : gzim@uni-hohenheim.de) 


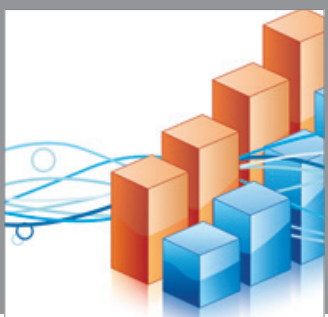

Advances in

Operations Research

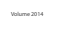

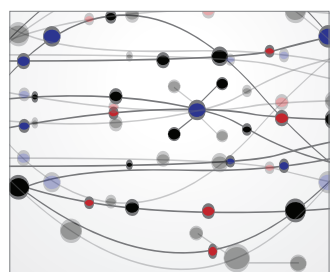

\section{The Scientific} World Journal
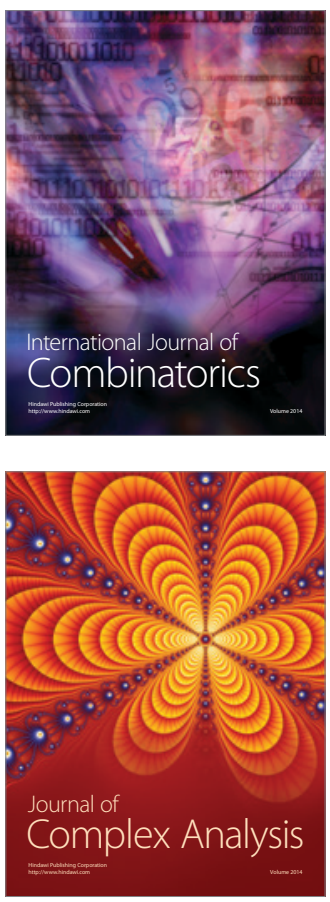

International Journal of

Mathematics and

Mathematical

Sciences
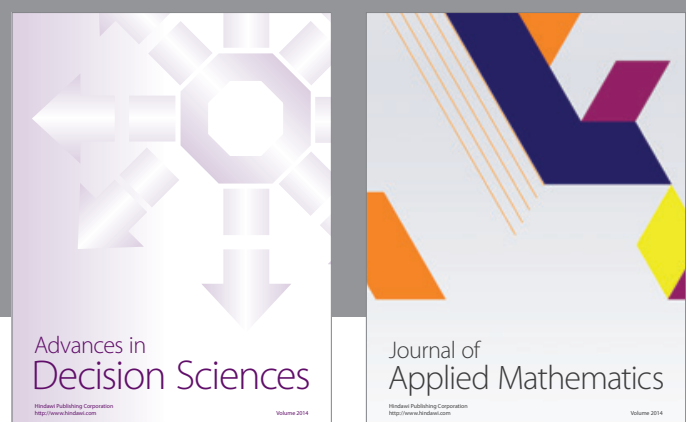

Journal of

Applied Mathematics
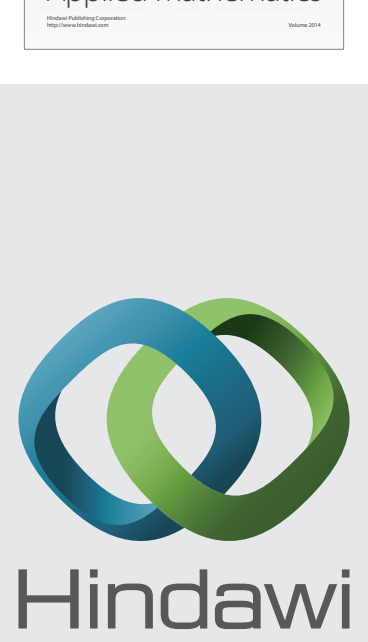

Submit your manuscripts at http://www.hindawi.com
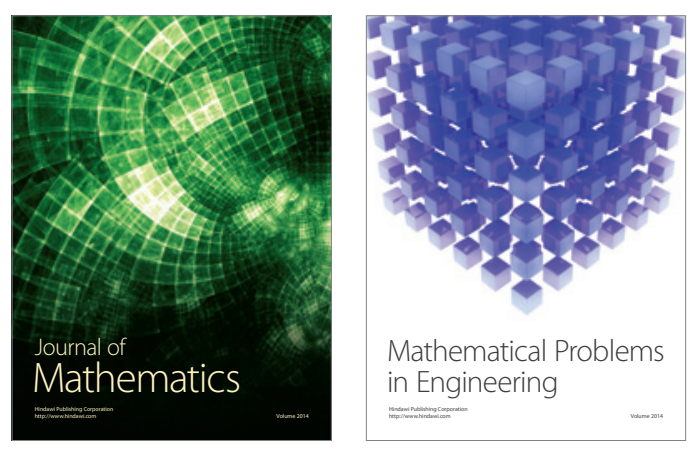

Mathematical Problems in Engineering
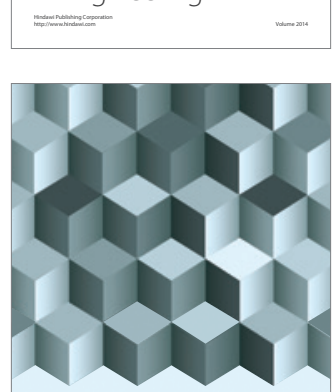

Journal of

Function Spaces
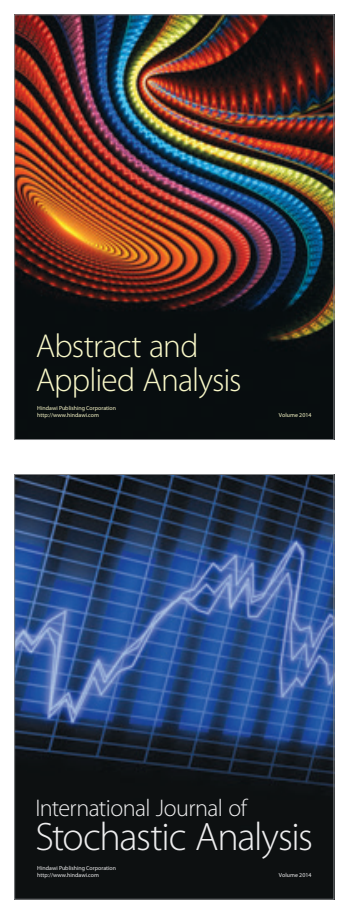

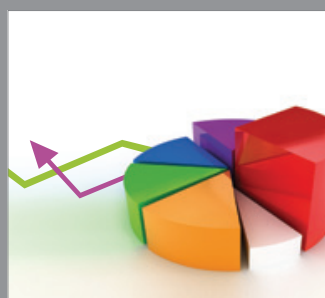

ournal of

Probability and Statistics

Promensencen
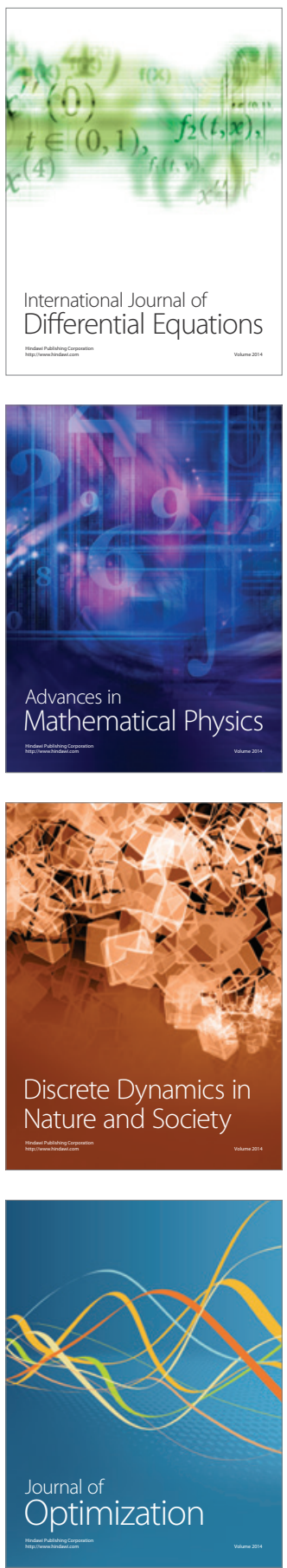